\title{
Saturation of electrical resistivity
}

\author{
O. Gunnarsson ${ }^{(1)}$, M. Calandra ${ }^{(2)}$ and J.E. Han ${ }^{(3)}$ \\ (1) Max-Planck-Institut für Festkörperforschung, Postfach 800665, D-70506 Stuttgart, Germany \\ (2) Laboratoire de Minéralogie-Cristallographie, case 115, 4 Place Jussieu, 75252, Paris cedex 05, France \\ (3) Department of Physics, The Pennsylvania State University, University Park, PA 16802-6300
}

\begin{abstract}
Resistivity saturation is observed in many metallic systems with large resistivities, i.e., when the resistivity has reached a critical value, its further increase with temperature is substantially reduced. This typically happens when the apparent mean free path is comparable to the interatomic separations - the Ioffe-Regel condition. Recently, several exceptions to this rule have been found. Here, we review experimental results and early theories of resistivity saturation. We then describe more recent theoretical work, addressing cases both where the Ioffe-Regel condition is satisfied and where it is violated. In particular we show how the (semiclassical) Ioffe-Regel condition can be derived quantum-mechanically under certain assumptions about the system and why these assumptions are violated for high- $T_{c}$ cuprates and alkali-doped fullerides.
\end{abstract}

\section{CONTENTS}

\author{
I. Introduction \\ II. Experimental results \\ III. Early theoretical work \\ IV. Calculation of resistivity \\ V. f-sum rule \\ VI. Weakly correlated broad band systems \\ VII. Strongly correlated systems. High- $T_{c}$ cuprates \\ VIII. Violation of the Ioffe-Regel condition in other \\ compounds \\ IX. Very large $T$ behavior. $\mathrm{C}_{60}$ compounds \\ X. Anderson metal-insulator transition and Mott's \\ minimum conductivity. \\ XI. Conclusions
}

Appendix A: Mean-free path

\section{INTRODUCTION}

The electrical resistivity, $\rho$, of metals is usually calculated in the Boltzmann theory, where the electrons are treated semiclassically. An electron is assumed to move with a wave vector $\mathbf{k}$ between scattering events, caused by phonons, other electrons, impurities or some other disorder. The average distance an electron moves between two scattering events is the mean free path $l$. Assuming a three-dimensional system and a spherical Fermi surface with one sheet, $\rho$ can be expressed in terms of $l$ as (see Appendix A)

$$
\rho=\frac{3 \pi^{2} \hbar}{e^{2} k_{F}^{2} l},
$$

where $k_{F}$ is the Fermi wave vector. Alternatively, if the resistivity is known experimentally, an apparent mean free path can be determined from Eq. (1). In the following, Eq. (1) will be used in the latter way to determine $l$, which then only depends on the experimental resistivity and the density of conduction electrons (giving $k_{F}$ ).

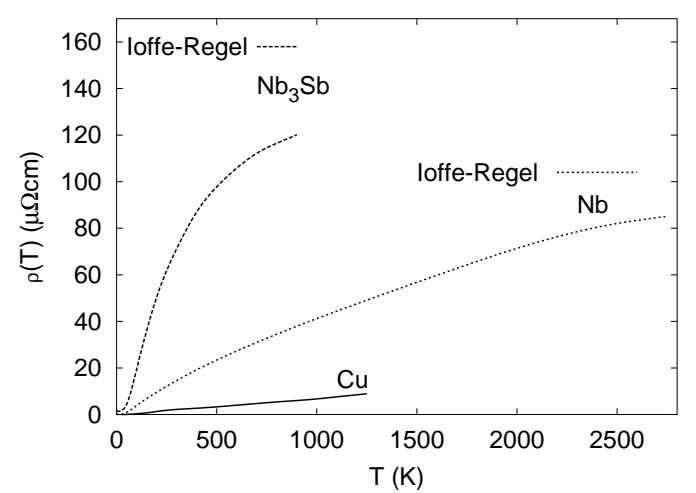

FIG. 1. Resistivity of $\mathrm{Cu}, \mathrm{Nb}_{3} \mathrm{Sb}$ (Fisk and Webb, 1976) and $\mathrm{Nb}$ (Abraham and Deviot, 1972). The figure also shows the the Ioffe-Regel (Ioffe and Regel, 1960) saturation resistivities of $\mathrm{Nb}_{3} \mathrm{Sb}$ and $\mathrm{Nb}$, setting the mean free path $l$ in Eq. (1) equal to the distance between the $\mathrm{Nb}$ atoms. The corresponding value for $\mathrm{Cu}, 260 \mu \Omega \mathrm{cm}$, falls outside the figure. The figure illustrates that for $\mathrm{Nb}_{3} \mathrm{Sb}$ and $\mathrm{Nb}$ the resistivity saturates roughly as predicted by the Ioffe-Regel criterion, while $\rho(T) \sim T$ for $\mathrm{Cu}$ at large $T$.

For a good metal like $\mathrm{Cu}, l$ is of the order of hundreds or even thousands of $\AA$. In the semiclassical Boltzmann theory and for $T$ larger than a fraction of a typical phonon energy, the resistivity due to phonon scattering grows linearly with $T$. This is shown for $\mathrm{Cu}$ in Fig. 1 . The apparent mean free path is then reduced correspondingly. For a good metal, however, $l$ is much larger than the separation $d$ of the atoms, even at the melting temperature.

For certain metals, in particular many transition metals and transition metal compounds, the resistivity behaves in a completely different way. This was emphasized by Fisk and Webb (1976), who studied the A15 compounds $\mathrm{Nb}_{3} \mathrm{Sb}$ (see Fig. 1). Similar results had earlier been obtained by Woodard and Cody (1964) for $\mathrm{Nb}_{3} \mathrm{Sn}$ and by several other groups for other compounds. For small $T, \rho(T)$ grows much faster than for $\mathrm{Cu}$ and $l$ be- 
comes comparable to $d$ already for $T$ of the order of several hundred K. At this point, the slope of the resistivity curve is substantially reduced, and $l$ stays comparable to $d$ for experimentally accessible values of $T$. This is referred to as resistivity saturation. It describes the situation where $\rho(T)$ grows much slower than $\rho(T) \sim T$, predicted by the Boltzmann equation, but it does not necessarily mean that $\rho(T)$ becomes a constant.

Ioffe and Regel (1960) pointed out that the semiclassical theory makes no sense if $l<d$, and we refer to $l \gtrsim d$ as the Ioffe-Regel condition. Inserting $l=d$ in Eq. (1) gives the Ioffe-Regel resistivity, shown in Fig. 1. In a semiclassical picture, it is natural that $l$ cannot be much smaller than $d$, since one may expect that an electron at most is scattered at every atom. Saturation is then expected when $l \sim d$. A semiclassical theory, however, breaks down when $l \sim d$, since the uncertainty in the $\mathbf{k}$-vector of an electron is comparable to the size of the Brillouin zone. A semiclassical theory cannot therefore explain saturation. Nevertheless, as discussed in Sec. II, in the 1970's and early 1980's a large number of metals were found which showed saturation when $l \sim d$, and the behavior seemed to be universal. During this time, much theoretical work was performed to explain saturation. No consensus was reached, however, and the interest turned to other problems.

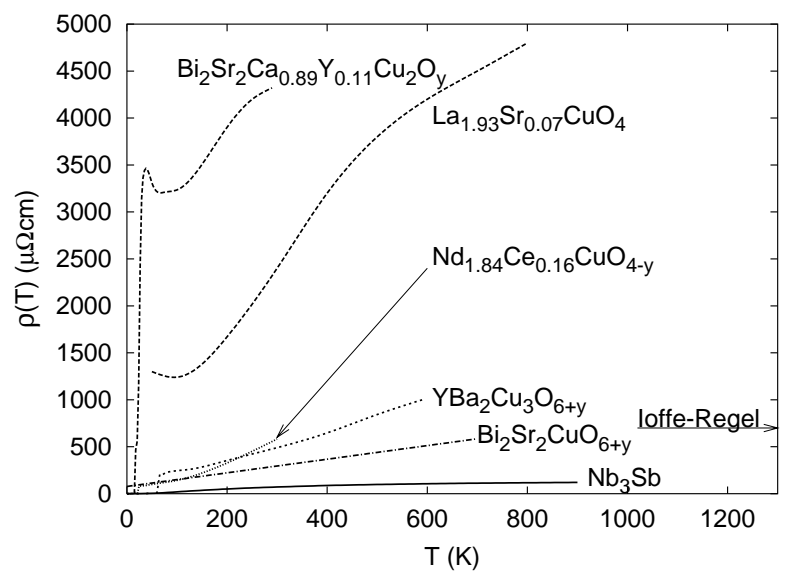

FIG. 2. Resistivity of $\mathrm{Bi}_{2} \mathrm{Sr}_{2} \mathrm{Ca}_{1-x} \mathrm{Y}_{x} \mathrm{Cu}_{2} \mathrm{O}_{8+y}\left(T_{c}=30\right.$ $\mathrm{K}$ ) (Wang et al., 1996ab), $\mathrm{La}_{1.93} \mathrm{Sr}_{0.07} \mathrm{CuO}_{4}$ (Takagi et al., 1992), $\mathrm{Nd}_{1.84} \mathrm{Ce}_{0.16} \mathrm{Cu}_{4-y}\left(T_{c}=22.5 \mathrm{~K}\right)$ (Hikada and Suzuki, 1989), $\mathrm{YBa}_{2} \mathrm{Cu}_{3} \mathrm{O}_{6+x}\left(T_{c}=60 \mathrm{~K}\right)$ (Orenstein et al., 1990), $\mathrm{Bi}_{2} \mathrm{Sr}_{2} \mathrm{Cu}_{6+y}\left(T_{c}=6.5 \mathrm{~K}\right)$ (Martin et al., 1990) and $\mathrm{Nb}_{3} \mathrm{Sb}$ (Fisk and Webb, 1976). The arrow shows the Ioffe-Regel resistivity of $\mathrm{La}_{1.93} \mathrm{Sr}_{0.07} \mathrm{CuO}_{4}$. The figure illustrates that there is no sign of saturation at the Ioffe-Regel resistivity, but in some cases perhaps at much larger resistivities. Observe the magnitude compared with $\mathrm{Nb}_{3} \mathrm{Sb}$.

The situation changed drastically in the late 1980's, when it was found that the high- $T_{c}$ cuprates behave very differently (Gurvitch and Fiory, 1987). Fig. 2 shows that the resistivity for these compounds is typically much larger than for metals as $\mathrm{Nb}_{3} \mathrm{Sb}$, satisfying the Ioffe-Regel condition. As described in Appendix A, the Ioffe-Regel resistivity for $\mathrm{La}_{2-x} \mathrm{Sr}_{x} \mathrm{CuO}_{4}$ is very large, about 0.7 $\mathrm{m} \Omega \mathrm{cm}$, due to the small carrier concentration. Nevertheless, the resistivities of the high- $T_{c}$ cuprates substantially exceed the Ioffe-Regel resistivity or are comparable to this resistivity without signs of saturation. The Ioffe-Regel condition is therefore strongly violated. Both $\mathrm{La}_{2-x} \mathrm{Sr}_{x} \mathrm{CuO}_{4}$ for small $x$ and $\mathrm{Bi}_{2} \mathrm{Sr}_{2} \mathrm{Ca}_{1-x} \mathrm{Y}_{x} \mathrm{Cu}_{2} \mathrm{O}_{8+y}$ show, however, signs of saturation, although at much larger values than the Ioffe-Regel resistivity. Fig. 3 shows other examples of the violation of the Ioffe-Regel condition, namely $\mathrm{Rb}_{3} \mathrm{C}_{60}$ (Hebard et al., 1993), $\mathrm{La}_{4} \mathrm{Ru}_{6} \mathrm{O}_{19}$ (Khalifah et al., 2001) and $\mathrm{Sr}_{2} \mathrm{RuO}_{4}$ (Tyler et al., 1998).

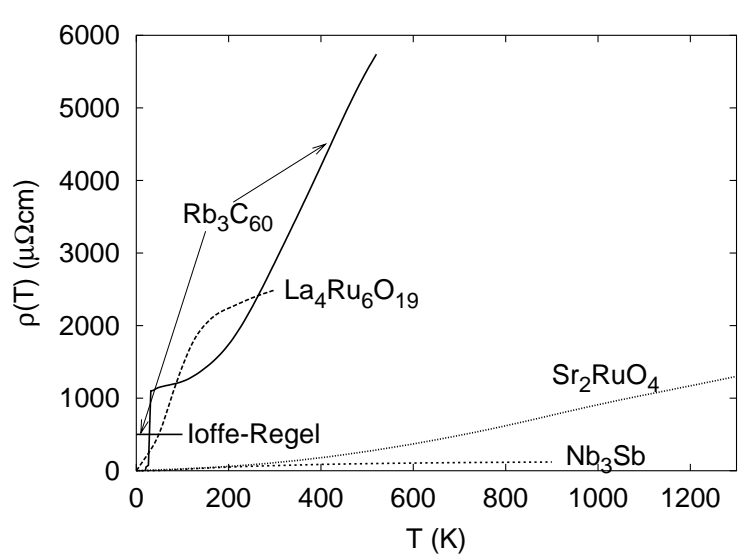

FIG. 3. Resistivity of $\mathrm{Rb}_{3} \mathrm{C}_{60}$ (Hebard et al., 1993) $\mathrm{La}_{4} \mathrm{Ru}_{6} \mathrm{O}_{19}$ (Khalifah et al., 2001), $\mathrm{Sr}_{2} \mathrm{RuO}_{4}$ (Tyler et al., 1998), $\mathrm{Nb}_{3} \mathrm{Sb}$ (Fisk and Webb, 1976) and the Ioffe-Regel resistivity for $\mathrm{Rb}_{3} \mathrm{C}_{60}$. There is no sign of saturation at the Ioffe-Regel resistivity, but $\mathrm{La}_{4} \mathrm{Ru}_{6} \mathrm{O}_{19}$ may saturate at a much larger resistivity.

Although there are substantial uncertainties in the absolute values of the experimental resistivity, it is, nevertheless, clear that the Ioffe-Regel condition can be violated. This shows that the semiclassical argument for saturation is not just questionable, but actually gives wrong predictions for these systems. This emphasizes the need for a theory of saturation going beyond the semiclassical treatment of the electrons. Such a theory should also explain the violation of the Ioffe-Regel condition for the systems mentioned above. The drastic change in the experimental situation over the last 15 years has led to a renewed interest in resistivity saturation.

In Sec. II, we review the experimental results, and in Sec. III early theoretical work. We then briefly describe methods for calculating the resistivity in Sec. IV. Much of the theoretical work has started from a Boltzmannlike approach, assuming that the scattering mechanism is a relatively weak perturbation. In Sec. V, we de- 
scribe an approach starting from the opposite limit of very strong scattering, assuming that the Drude peak has been completely removed. This approach is based on the f-sum rule. It leads to an approximate upper limit $\rho_{\text {sat }}$ to the resistivity, which usually has a weak $T$ dependence. Resistivity saturation then happens if the initial slope of $\rho(T)$ is so large that $\rho_{\text {sat }}$ is reached at small values of $T$ and if $\rho_{\text {sat }}$ has a weak $T$ dependence. This approach is applied to three models with different saturation behavior. In Sec. VI, we describe the treatment of a model of weakly correlated electrons in a broad band, appropriate for many transition metal compounds. This model shows saturation in agreement with the Ioffe-Regel condition. We show how this condition can be derived quantum-mechanically by assuming i) noninteracting electrons and ii) $T \ll W$, where $W$ is the band width. In Sec. VII we treat high- $T_{c}$ cuprates, for which assumption i) about noninteracting electrons is violated. Using the $t-J$ model, we find that saturation can occur, but at much larger resistivities than predicted by the Ioffe-Regel condition. Other metals violating the Ioffe-Regel condition are discussed in Sec. VIII. In Sec. IX, we describe a model of alkali-doped $\mathrm{C}_{60}$, which shows no saturation. This result depends on the intramolecular character of the phonons and on condition ii) $(T \ll W)$ being violated. The relation to Anderson localization and Mott's minimum conductivity is discussed in Sec. X.

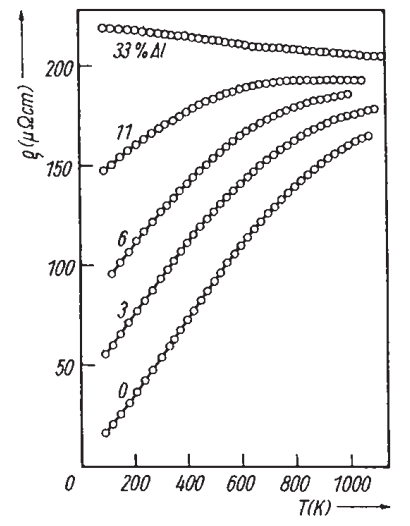

FIG. 4. Resistivity of $\mathrm{Ti}_{1-x} \mathrm{Al}_{x}$ alloys. The figure suggests that the saturation resistivity is independent of the disorder (after Mooij, 1973).

\section{EXPERIMENTAL RESULTS}

Most cases of resistivity saturation have been found for transition metal compounds. Mooij (1973) made one of the first observations for $\mathrm{Ti}_{1-x} \mathrm{Al}_{x}$ (see Fig. 4) and several other alloys. In addition, he found that the positive $T$ dependence of the resistivity becomes weaker or even negative for strongly disordered systems. Similar results were found by Arko et al. (1973) and by Tsuei (1986) for other compounds. These results suggest that compo- sitional disorder and disorder due to thermally excited phonons can have a similar effect. Other early examples were found by Fisk and Lawson (1973).

As discussed in the introduction, resistivity saturation was found for A15 compounds (Woodard and Cody, 1964; Marchenko, 1973; Fisk and Webb, 1976; Wiesmann et al., 1977; Gurvitch et al., 1978). Another class of systems showing resistivity saturation is the Chevrel phases (Martin et al., 1978; Sunandana, 1979). Early measurements gave very large values for the resistivity, probably because of sample problems, but later measurements on single crystals gave resistivities comparable to those of A15 compounds (Kitazawa et al., 1981). Fig. 5 shows the resistivity of the $3 \mathrm{~d}$ and $5 \mathrm{~d}$ transition metals. Several of these metals show clear signs of saturation, in particular $\alpha$-Mn. Other cases are less clear, and, e.g., the resistivity of W even bends slightly upwards, although it is already large. As discussed at the end of this section, $\mathrm{W}$ is nevertheless an example of saturation, if anharmonic effects are considered. The above metals can be considered as cases where the Ioffe-Regel condition is satisfied.
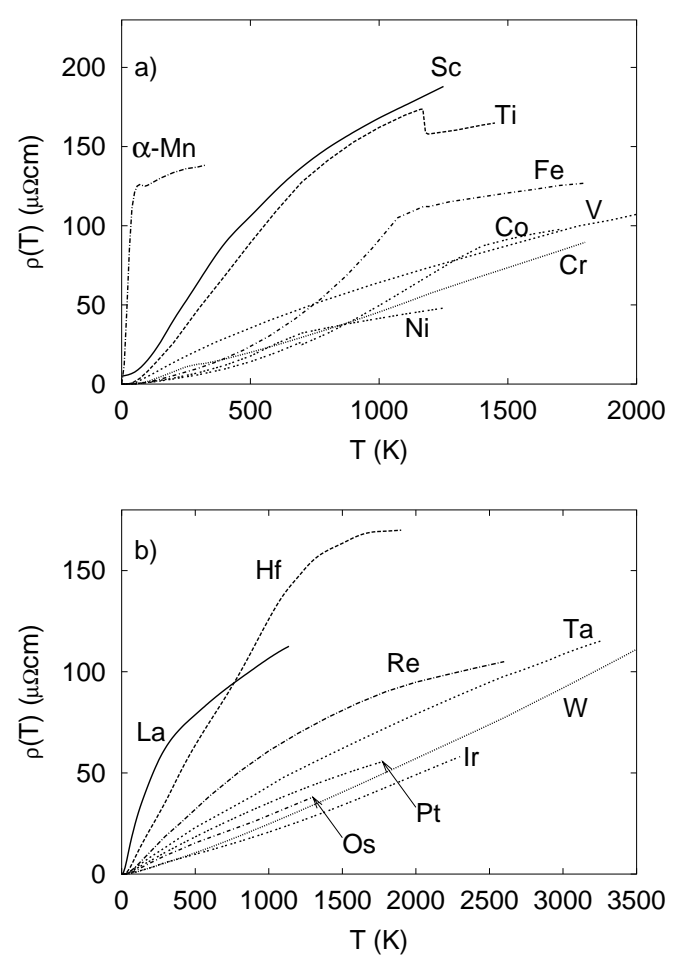

FIG. 5. Resistivity of 3d (a) and 5d (b) transition metals (Bass, 1982).

We next consider the in-plane resistivity of high- $T_{c}$ cuprates. The resistivity depends strongly on the doping of the $\mathrm{CuO}_{2}$ plane. Information about the doping is given by the superconductivity transition temperature $T_{c}$, which is quoted below, when available. As the doping is reduced below the optimum value, $T_{c}$ drops and $\rho$ 
increases. In the case of $\mathrm{La}_{2-x} \mathrm{Sr}_{x} \mathrm{CuO}_{4}$ we assume that the doping is given by $x$. Results for several cuprates were given in Fig. 2, and we give some further examples in Table I. These are examples of systems where the resistivity is comparable to the Ioffe-Regel resistivity already at moderate values of $T$ and without showing signs of saturation.

TABLE I. Resistivity $\rho(T)$ (in $\mathrm{m} \Omega \mathrm{cm}$ ) of high- $T_{c}$ cuprates. The measurment temperature $T$ and the superconductivity transition temperature $T_{c}$ are given in $\mathrm{K}$.

\begin{tabular}{lllll}
\hline \hline Compound & $T_{c}$ & $T$ & $\rho(T)$ & Reference \\
\hline $\mathrm{HgBa}_{2} \mathrm{Ca}_{0} \mathrm{Cu}_{1} \mathrm{O}_{4+x}$ & 94 & 300 & 0.5 & Daignere et al., 2001 \\
$\mathrm{HgBa}_{2} \mathrm{Ca}_{1} \mathrm{Cu}_{2} \mathrm{O}_{6+x}$ & 122 & 300 & 0.3 & Yan et al., 1998 \\
$\mathrm{HgBa}_{2} \mathrm{Ca}_{2} \mathrm{Cu}_{3} \mathrm{O}_{8+x}$ & 125 & 500 & 0.6 & Carrington et al., 1994 \\
$\mathrm{HgBa}_{2} \mathrm{Ca}_{3} \mathrm{Cu}_{4} \mathrm{O}_{10+x}$ & 130 & 400 & 0.5 & Löhle et al., 1996 \\
$\mathrm{Tl}_{2} \mathrm{Ba}_{2} \mathrm{CuO}_{6+y}$ & 80 & 300 & 1.3 & Kubo et al., 1991 \\
$\mathrm{Tl}_{2} \mathrm{Ba}_{2} \mathrm{CuO}_{6+y}$ & 80 & 270 & 0.6 & Duan et al., 1991 \\
$\mathrm{TlSr}_{2} \mathrm{CaCu}_{2} \mathrm{O}_{7-y}$ & 65 & 300 & 0.5 & Kubo et al., 1991 \\
$\mathrm{Bi}_{2} \mathrm{Sr}_{2} \mathrm{CaCu}_{2} \mathrm{O}_{8+y}$ & 76 & 300 & 1.2 & Chen et al., 1998 \\
\hline \hline
\end{tabular}

$\mathrm{Bi}_{2} \mathrm{Sr}_{2} \mathrm{Ca}_{1-x} \mathrm{Y}_{x} \mathrm{Cu}_{2} \mathrm{O}_{8+y}$ is of particular interest, since Wang et al. (1996ab) found saturation for the most underdoped samples, e.g., $x=0.11\left(T_{c}=30 \mathrm{~K}\right)$ as shown in Fig. 2. Similar results have also been obtained by Forro et al. (2002). Other measurements only showed weak signs of saturation (Mandrus et al., 1992; Ruan et al., 2001). In the measurements of Ruan et al. (2001), the reason may have been that their $x=0.11$ sample had a higher $T_{c}=56 \mathrm{~K}$ than that of Wang et al. (1996ab) $\left(T_{c}=30 \mathrm{~K}\right)$, suggesting that the former sample was less underdoped. We conclude that the Ioffe-Regel condition is violated for high- $T_{c}$ superconductors but that some systems with very large resistivities may show signs of saturation at much larger values than the Ioffe-Regel resistivity.

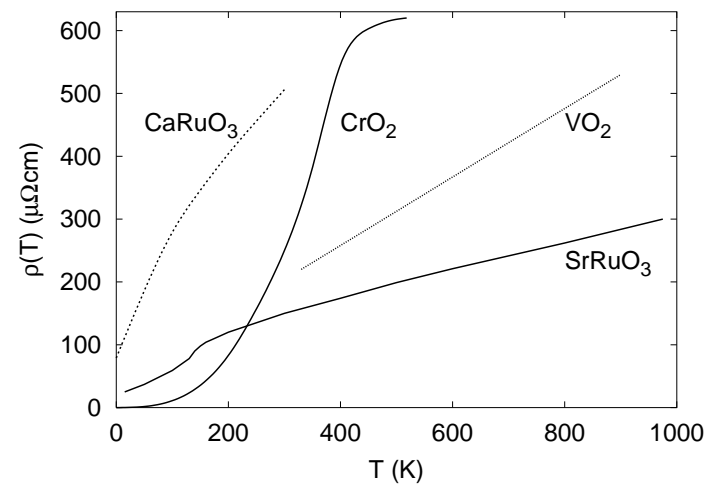

FIG. 6. Resistivity of $\mathrm{CaRuO}_{3}$ (Klein et al., 1999ab), $\mathrm{CrO}_{2}$ (Rodbell et al., 1966), $\mathrm{VO}_{2}$ (Allen et al., 1993) and $\mathrm{SrRuO}_{3}$ (Allen et al., 1996).

Apart from the high- $T_{c}$ cuprates, a number of tran- sition metal compounds have been found which violate the Ioffe-Regel condition. Fig. 3 and Fig. 6 shows some examples. Some of these metals show signs of saturation $\left(\mathrm{La}_{4} \mathrm{Ru}_{6} \mathrm{O}_{19}, \mathrm{CrO}_{2}\right.$ and perhaps $\left.\mathrm{CaRuO}_{3}\right)$, but at higher resistivities than the Ioffe-Regel resistivity, while other systems show no sign of saturation. Several of theses metals are believed to be non-Fermi liquids. The data in Figs. 3 and 6 were obtained from single crystals, except for $\mathrm{Rb}_{3} \mathrm{C}_{60}$ and $\mathrm{CaRuO}_{3}$. This could be a reason why the resistivity in Fig. 6 is much larger for $\mathrm{CaRuO}_{3}$ than for the related $\mathrm{SrRuO}_{3}$. Large resistivities are also found in manganites (Salamon and Jaime, 2001). These systems raise interesting problems in terms of the colossal magnetoresistance, possibly important polaron effects and many phase transitions, but they are outside the scope of this paper.

The resistivity of $\mathrm{A}_{3} \mathrm{C}_{60}(\mathrm{~A}=\mathrm{K}, \mathrm{Rb})$ shows a quite substantial spread between different measurements (Hebard et al., 1993; Hou et al., 1993; Palstra et al., 1994; Degiorgi et al, 1994). These have been performed for thin films and doped single crystals using direct and optical methods. In particular the thin films may have substantial defects (e.g., grain boundaries) which would increase the resistivity. The optical measurements should, however, be much less sensitive to this. Indeed, optical measurements for doped single crystals (Degiorgi et al., 1994) gave resistivities that are about a factor of two smaller than those obtained from direct measurements for thin films (Palstra et al., 1994), but comparable to (somewhat larger than) direct measurements for doped single crystals (Hou at al., 1993). This suggests that the thin film results in Fig. 3 could overestimate the resistivity of $\mathrm{Rb}_{3} \mathrm{C}_{60}$ by a factor of two or somewhat more. This would not change the qualitative conclusion that the Ioffe-Regel condition is violated for $\mathrm{A}_{3} \mathrm{C}_{60}$.

Experiments for $\mathrm{Rb}_{3} \mathrm{C}_{60}$ show no signs of saturation up to about $500 \mathrm{~K}$ (Hebard et al., 1993). It is difficult to reach higher values of $T$, due to the possibility of thermally driven rearrangements of the alkali atoms. Hou et al. (1995), using a pulsed heating technique to reach 800 $\mathrm{K}$, found a small change in the slope at about $T=500 \mathrm{~K}$. This was interpreted as a sign of saturation. Using the parallel resistor formula (Wiesmann et al., 1977), they deduced the saturation resistivity $6 \pm 3 \mathrm{~m} \Omega \mathrm{cm}$, corresponding to the mean free path $l=1 \pm 0.5 \AA$. Thus there is no saturation at $l \sim d$, where $d=10 \AA$ is the separation of two $\mathrm{C}_{60}$ molecules (see Appendix A), but it is hard to judge if there is saturation at some larger resistivity.

The Boltzmann equation predicts $\rho(T) \sim T$ for large $T$ only if various parameters, such as the electron-phonon interaction, are independent of $T$. This assumption is sometimes strongly violated. For instance, anharmonic effects reduce the phonon frequencies of $\mathrm{W}$ by about 30 $\%$ at the melting point (Grimvall, 2001; Grimvall et al., 1987; Guillermet and Grimvall, 1991). According to the 
Boltzmann equation, this should increase $\rho(T)$ by about a factor of two, due to an increase in the electron-phonon coupling, giving a growth of $\rho(T)$ which is much faster than linear. Experimentally, however, the growth is only slightly faster than linear (Fig. 5b). This suggests that saturation in $\mathrm{W}$ is masked by the reduction of the phonon frequencies (Grimvall, 2001).

The resistivity is usually measured at constant pressure. As $T$ is increased, the crystal expands and various parameters are changed. Sundqvist and Andersson (1990) converted the resistivity of $\mathrm{YBa}_{2} \mathrm{Cu}_{3} \mathrm{O}_{7-y}$ to constant volume data, which is the more relevant quantity for theoretical discussions. They found that this lowered the $T=500 \mathrm{~K}$ resistivity by about $32 \%$. Although this conversion involves many uncertainties (Sundqvist and Andersson, 1990), it was concluded that the pronounced linearity in the constant pressure data is lost. Actually, the corrected data show signs of saturation. A similar correction of the data for $\mathrm{Rb}_{3} \mathrm{C}_{60}$ (Vareka and Zettl, 1994) changed the approximately quadratic $T$ dependence found for constant pressure (see Fig. 3) to an approximately linear dependence for constant volume.

\section{EARLY THEORETICAL WORK}

Resistivity saturation was studied theoretically very intensively from the middle of the 1970's to the early 1980 's. In this section we review some of this work as well as some later work. We focus on work related to the electron-phonon scattering, while work based on electron-electron scattering (Jarrell and Pruschke, 1994; Lange and Kotliar, 1999; Parcollet and Georges, 1999; Merino and McKenzie, 2000; Gunnarsson and Han, 2000) fall outside the scope of this review.

Much of the early work has been reviewed by Allen (1980ab). Initially, explanations were proposed in terms of strong variations of the electronic structure on the energy scale of $k_{B} T$ or unusual anharmonic effects. These effects appear, however, to be too small to explain experiment, and as ever more examples of resistivity saturation were discovered, such theories had to be abandoned (Allen, 1980ab).

Wiesmann et al. (1977) found empirically that the resistivity of systems such as $\mathrm{Nb}_{3} \mathrm{Sb}$ can be rather accurately described by a parallel resistor formula

$$
\frac{1}{\rho(T)}=\frac{1}{\rho_{\text {sat }}}+\frac{1}{\rho_{\text {ideal }}(T)},
$$

where $\rho_{\text {sat }}$ is a saturation resistivity and $\rho_{\text {ideal }}(T)$ is the resistivity of the Boltzmann equation, i.e., linear in $T$ for large $T$.

Mooij (1973) observed that strong disorder can lead to a negative slope of $\rho(T)$ (Fig. 4). Jonson and Girvin (1979), Girvin and Jonson (1980) and Imry (1980) argued that this is due to an incipient Anderson transition.
The disorder is assumed to be so large that the system is close to an Anderson transition for $T=0$. As $T$ is increased, inelastic scattering (phonon assisted hopping) leads to a loss of phase information (Lee and Ramakrishnan, 1985) and the system moves away from the Anderson transition. This was argued to lead to a reduction of the resistivity, as observed by Mooij (1973) for strongly disordered systems.

Weger and Mott (1985) and Weger (1985) proposed a theory in terms dehybridization of the $s$ - and $d$-electrons at large values of $T$. Laughlin (1982) presented a theory where exchange interaction reduced the density of states and increased the Fermi velocity in such a way that resistivity saturation was obtained.

Cote and Meisel (1978) and Morton et al. (1978) proposed that an electron is only scattered by a phonon if the mean free path of the electron is longer than the phonon wave length. As $T$ is increased and $l$ becomes shorter, an increasing number of phonons become inefficient scatters. With these assumptions, resistivity saturation can be derived. Later calculations treating the electrons quantum mechanically, as described before Eq. (5), do not seem to support the basic assumption in this theory (Calandra and Gunnarsson, 2002).

Several theories have been based on models with one electronic state per unit cell. Christoph and Shiller (1984) studied the Fröhlich Hamiltonian. Using a truncated equation of motion approach, they included some higher order terms in the electron-phonon interaction. In this way they derived the parallel resistor formula (Eq. (2)). As pointed out by them, their $\rho_{\text {sat }} \sim 0.84 \mathrm{~m} \Omega \mathrm{cm}$ is substantially larger than what is observed for transition metal compounds, but it is comparable to what might be expected for a one band model, as in Eq. (11) below $\left(N_{d}=1\right)$. Belitz and Schirmacher (1983) studied a generalized Frölich model which also included impurity scattering. They included higher order terms by using a mode coupling technique in a memory function approach. They found a rather good agreement with experiment, but a substantially smaller $\rho_{\text {sat }}$ than might have been expected from Eq. (11) for $N_{d}=1$.

Ron et al. (1986) treated a Kronig-Penney chain, where the positions of the $\delta$-functions vibrate. Using the Landauer formula they found saturation in the limit of large amplitude vibrations. Their $\rho_{\text {sat }}$ depends, however, on the strength of the potentials, and it therefore appears that the Ioffe-Regel condition is not explained.

The Boltzmann equation only includes intraband transitions. Chakraborty and Allen (1979) and Allen and Chakraborty (1981) emphasized that resistivity saturation is typically found for systems where interband transitions are important. They included these transitions by generalizing the Boltzmann equation, treating terms of the order $(d / l)^{0}$, while the Boltzmann equation only 
contains terms of the of the order $(d / l)^{-1}$. They demonstrated that this leads to a parallel resistor type of formula (Eq. (2)) and estimated the saturation resistivity to be of the right order of magnitude. Due to the complexity of the equations, it was not possible to make explicit calculations and the sign of the $\rho_{\text {sat }}$ in Eq. (2) could not be determined. They also pointed out that higher order terms in $(d / l)$ may be important.

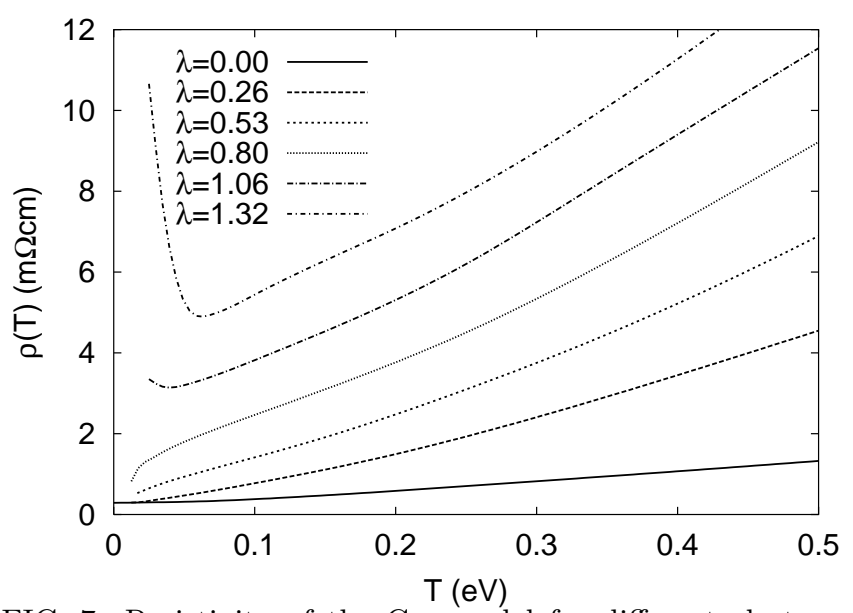

FIG. 7. Resistivity of the $\mathrm{C}_{60}$ model for different electronphonon couplings $\lambda$ according to QMC calculations (Gunnarsson and Han, 2000; Calandra and Gunnarsson, 2002). The phonon frequency is $\omega_{p h}=0.1 \mathrm{eV}$. The figure illustrates the lack of saturation. For $\lambda=0.80$ the onset of superconductivity can be seen as a sharp downturn in $\rho(T)$ as $T$ is lowered, due to superconducting fluctuations. For $\lambda=1.06$ and 1.32, the resistivity has a negative slope for small $T$, indicating an insulating system.

Millis et al. (1999) studied a Holstein like model, where the phonons couple to the electron level energies (in the following called LE coupling). They found that there is a change of slope in the $\rho(T)$ curve for the case of a strong electron-phonon coupling, and associated this with resistivity saturation. They observed, however, that the resistivity does not saturate and remarked that resistivity saturation is a misnomer. Gunnarsson and Han (2000) performed a quantum Monte-Carlo (QMC) calculation for a somewhat related model of $\mathrm{A}_{3} \mathrm{C}_{60}(\mathrm{~A}=\mathrm{K}$, $\mathrm{Rb})$. This model included the partly occupied, threefold degenerate $t_{1 u}$ level as well as the LE coupling to a five-fold degenerate $\mathrm{H}_{g}$ phonon. We refer to this as the $\mathrm{C}_{60}$ model. The results, shown in Fig. 7, are somewhat similar to those of Millis et al. (1999), but they were considered as an example of lack of saturation. It was later shown (Calandra and Gunnarsson, 2001) that a more realistic description of saturation can be obtained by using a coupling to the hopping integrals (HI coupling) (see Sec. VI).

\section{CALCULATION OF RESISTIVITY}

The resistivity is usually calculated in the Boltzmann theory. The electrons are treated semiclassically, and are assumed to be accelerated by the electric field between scattering processes, caused by impurities, phonons or other electrons. This can be considered as the lowest order theory in an expansion in $1 /\left(k_{F} l\right)$ (Kohn and Luttinger, 1957). Both because of this and because of its prediction $\rho(T) \sim T$ for large $T$, it is clear that the Boltzmann equation cannot be used for describing saturation. Instead, it is necessary to treat the electrons quantummechanically. This can be done in the Kubo formalism. For an isotropic system, this requires the calculation of a current-current correlation function (Mahan, 1990)

$$
\pi(i \omega)=-\frac{1}{3 N \Omega} \int_{0}^{\beta} d \tau e^{i \omega \tau}\left\langle T_{\tau} \hat{\mathbf{j}}(\tau) \cdot \hat{\mathbf{j}}(0)\right\rangle
$$

where $N$ is the number of atoms, $\Omega$ is the volume per atom, $\beta=1 /\left(k_{B} T\right), k_{B}$ is the Boltzmann constant, $T_{\tau}$ is a time-ordering operator and $\mathbf{j}$ is the current operator. $\pi(i \omega)$ is analytically continued to real frequencies, giving $\pi_{\text {ret }}(\omega)$. The optical conductivity is then

$$
\sigma(\omega)=-\frac{\operatorname{Im} \pi_{\mathrm{ret}}(\omega)}{\omega}
$$

and the resistivity is $1 / \sigma(0)$.

$\pi(i \omega)$ can be calculated for imaginary times by using a determinantal quantum Monte-Carlo (QMC) method (Blankenbecler et al., 1981). It is then analytically continued to real frequencies by using a maximum entropy method (Jarrell and Gubernatis, 1996). This approach is very useful for establishing the saturation properties of a given model.

To interpret the results, a simpler method is used, where the phonons are treated semiclassically (Calandra and Gunnarsson, 2001; 2002). A large supercell with $N$ atoms and periodic boundary conditions is considered. Each phonon coordinate is given a random displacement according to a Gaussian distribution with the width determined by $T$. This set of displaced coordinates (snap shot) defines a new Hamiltonian. While the original Hamiltonian represents a complicated many-body problem due to the electron-phonon interaction, the dynamics of the phonons have been eliminated in the new Hamiltonian. Since we assume no electron-electron interaction, this is then a one-particle Hamiltonian for a disordered system. The corresponding eigenstates $|l\rangle$ and eigenvalues $\varepsilon_{l}$ are found. The Kubo-Greenwood formula (Greenwood, 1958) is then used

$$
\sigma_{x x}(\omega)=\frac{2 \pi}{N \Omega \omega} \sum_{l l^{\prime}}\left|\left\langle l\left|\hat{j}_{x}\right| l^{\prime}\right\rangle\right|^{2}\left(f_{l}-f_{l^{\prime}}\right) \delta\left(\hbar \omega-\varepsilon_{l^{\prime}}+\varepsilon_{l}\right),
$$


where $\hat{j}_{x}$ is the current operator in the $x$ direction, and $f_{l}$ is the Fermi function for the energy $\varepsilon_{l}$. It is essential that in this approach only the phonons are treated semiclassically and that the electrons are treated quantummechanically. This is in contrast to the semiclassical treatment of the electrons in the Boltzmann theory, which is unable to describe saturation.

In the following sections we discuss a new theory for describing different classes of materials with different saturation properties (Gunnarsson and Han, 2000; Calandra and Gunnarsson, 2001; 2002; 2003).

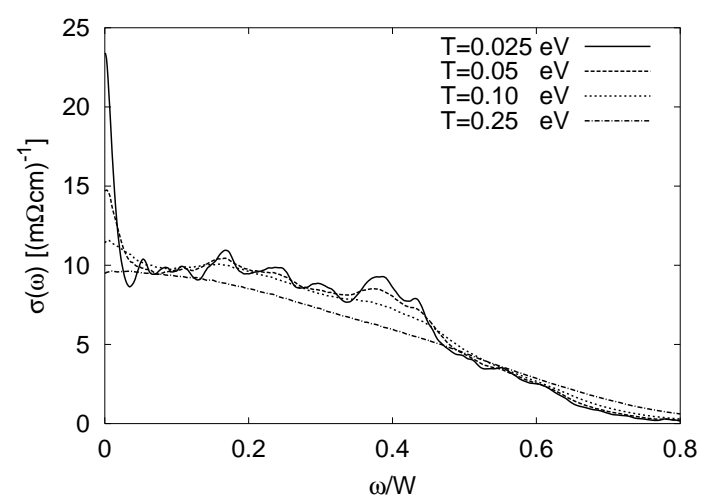

FIG. 8. $\sigma(\omega)$ as a function of $\omega$ for the $\mathrm{Nb}_{3} \mathrm{Sb}$ model in the semiclassical treatment of the phonons. The frequency has been scaled by the $T=0$ band width $W$. The figure illustrates how the Drude peak is lost as $T$ is increased.

\section{V. f-SUM RULE}

For small $T$, the optical conductivity $\sigma(\omega)$ typically has a Drude peak, due to intraband transitions between quasiparticles and centered at $\omega=0$, and a remaining incoherent part, as illustrated in Fig. 8 for $\mathrm{Nb}_{3} \mathrm{Sb}$. As $T$ is increased, the height of the Drude peak is reduced, and for large $T$ it disappears. This is also illustrated in Fig. 9, which shows the experimental optical conductivity of $\mathrm{CrO}_{2}$ at $T=300 \mathrm{~K}$. The arrow marks the conductivity corresponding to the saturation resistivity (see Fig. 6). This conductivity is similar to the magnitude of the incoherent contribution (at $300 \mathrm{~K}$ ). Again, this suggests that as $T$ is increased, the Drude peak disappears without the incoherent part changing very much. In the following, we therefore assume that $T$ is so large that the Drude peak has disappeared (Calandra and Gunnarsson, 2002).

The Boltzmann theory starts from the periodic system and treats the scattering mechanisms as small perturbations. The theory focuses on intraband transitions and the Drude peak. Here we start from the the opposite limit, where the scattering is so strong that the Drude peak disappears.

To estimate the incoherent contribution to $\sigma(\omega)$, we use the f-sum rule. We focus on tight-binding models with one type of electrons, e.g., $d$ electrons in a transition metal compound. Then there are no on-site matrix elements of the current operator. The off-site matrix elements can be related to hopping matrix elements, using charge and current conservation. For an isotropic threedimensional system, the tight-binding version of the $\mathrm{f}$ sum rule then takes the form (Maldague, 1977; Calandra and Gunnarsson, 2002)

$$
\int_{0}^{\infty} \sigma(\omega) d \omega=\frac{\pi}{6} \frac{d^{2} e^{2}}{N \Omega \hbar^{2}}\left|E_{K}\right|
$$

where $E_{K}$ is the hopping (kinetic) energy, $N$ is the number of atoms and $\Omega$ is the volume per atom. For a system with just nearest neighbor hopping and fixed atoms, $d$ is the separation of the sites. If the atoms vibrate or if there is hopping between more distant neighbors, $d$ is a weighted average of neighboring distances (Calandra and Gunnarsson, 2002).

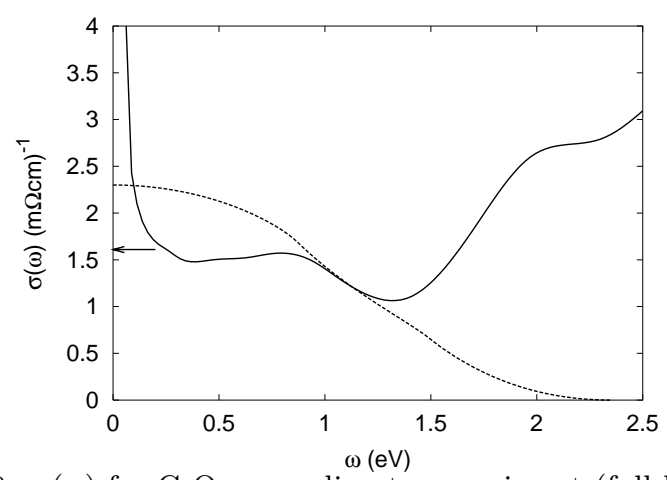

FIG. 9. $\sigma(\omega)$ for $\mathrm{CrO}_{2}$ according to experiment (full line) at $T=300 \mathrm{~K}$ (Singley et al., 1999) and the large $T$ model in Fig. 10 (dotted line), adjusting the area to the sum rule (6). The experimental intensity above $\omega \sim 1.5 \mathrm{eV}$ is due to interband transitions. The arrow shows the conductivity corresponding to the saturation resistivity in Fig. 6. It suggests that for large $T$, the Drude peak in the experimental $\sigma(\omega)$ disappears.

For models with only one type of electrons, e.g, $d$ electrons, $\sigma(\omega)$ at large $T$ looks schematically as in Fig. 10. This can be compared with the calculation for $\mathrm{Nb}_{3} \mathrm{Sb}$ in Fig. 8 or the expected large $T$ contribution from the $t_{2 g}$ band of $\mathrm{CrO}_{2}$ in Fig. 9 (Drude peak gone), excluding the interband transitions for $\hbar \omega>1.5 \mathrm{eV}$. For $\hbar \omega$ larger than the band width $W, \sigma(\omega) \approx 0$. If $\sigma(\omega)$ furthermore lacks large structures around $\omega=0$, we can approximate

$$
\sigma(\omega)= \begin{cases}\sigma(0) / \gamma, & \text { if } \hbar|\omega| \leq W \\ 0, & \text { otherwise }\end{cases}
$$

Then $\int \sigma(\omega) \hbar d \omega=\sigma(0) W / \gamma$, and the f-sum rule (6) requires that $\sigma(0)$ is given by the right hand side of Eq. (6) multiplied by $\gamma \hbar / W$ (Calandra and Gunnarsson, 2002; 2003), i.e., 


$$
\sigma(0)=\frac{\gamma \hbar}{W} \int_{0}^{\infty} \sigma(\omega) d \omega=\frac{\pi \gamma}{6 W} \frac{d^{2} e^{2}}{N \Omega \hbar}\left|E_{K}\right|
$$

where $\gamma \sim 2$ depends on the shape of $\sigma(\omega)$. This provides an approximate lower limit to the conductivity (upper limit to the resistivity), which may be reached if $T$ is so large that the Drude peak is negligible. The conductivity could be appreciably smaller only if there is a substantial amount of weight for $\omega>W$ or if $\sigma(\omega)$ is anomalously small for $\omega=0$. The former should not be important, since $\hbar \omega>W$ requires multiple electron-hole pair excitations. Since the current operator is a one-particle operator, these excitations should have a small weight. The latter could happen in the case of an incipient Anderson transition, but it should normally not occur at large $T$, where inelastic scattering destroys the phase information required for an Anderson localization (Lee and Ramakrishnan, 1985). The (lower) limit (8) to the conductivity is $T$ dependent, due to the $T$ dependence of $E_{K}$ and $W$. This $T$ dependence is often, but not always, rather weak. Below we use Eq. (8) to discuss different classes of metals.

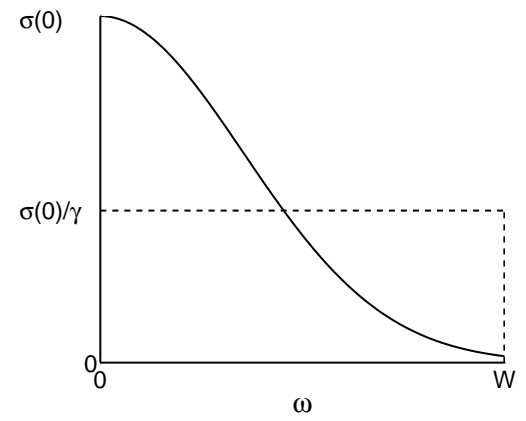

FIG. 10. Schematic picture of $\sigma(\omega)$. The average of $\sigma(\omega)$ over the band width is given by $\sigma(0) / \gamma$, where $\gamma \sim 2$.

\section{WEAKLY CORRELATED BROAD BAND SYSTEMS}

We first focus on metals with a large band width $W$. We therefore assume that i) $W$ is so large that the electrons can be treated as noninteracting and ii) $T \ll W$. In particular, we focus on $\mathrm{Nb}_{3} \mathrm{Sb}$, showing a pronounced saturation, and $\mathrm{Nb}$, showing a much weaker saturation (see Fig. 1). We use a model with an $N_{d}$-fold degenerate orbital on each site. The orbitals on different atoms are connected by hopping matrix elements, $t_{i m, j m^{\prime}}$, where $i$ and $j$ label the atoms and $m, m^{\prime}=1, . ., N_{d}$ the orbital. Then the electronic Hamiltonian is

$$
H^{\mathrm{el}}=\sum_{i \neq j, m m^{\prime} \sigma} t_{i m, j m^{\prime}} c_{i m \sigma}^{\dagger} c_{j m^{\prime} \sigma},
$$

Three Einstein phonons are introduced for each atom, describing its displacement in the three coordinate directions. The phonons couple to the hopping integrals, since these depend on the atomic positions. We refer to this as HI coupling and the model is referred to as the transition metal (TM) model. The model for $\mathrm{Nb}$ has $4 d$ orbitals $\left(N_{d}=5\right)$ on a bcc lattice. For $\mathrm{Nb}_{3} \mathrm{Sb}$ we use the $\mathrm{Nb}_{3}^{*}$ model, neglecting the Sb atoms (Pickett et al., 1979), and putting $4 d$ orbitals on the $\mathrm{Nb}$ sites. The models are therefore identical except for the lattice structure. For strongly ionic systems, the coupling to the level energies (LE) may also be important.

Fig. 11 shows the QMC (circles) results for the resistivity of $\mathrm{Nb}_{3}^{*}$ model for a supercell with $N=36$ atoms. These results extrapolate to a large resistivity at $T=0$. On the other hand, the resistivity of the model must be zero at $T=0$. It then follows that there must be a large change of the slope of $\rho(T)$ as $T$ is increased, i.e., resistivity saturation. This model can therefore be used for analyzing saturation. Fig. 11 also shows that the semiclassical treatment of the phonons is quite accurate for the TM model, and that it therefore can be used for interpreting the results.

To use f-sum rule of Sec. V, we calculate the hopping energy $E_{K}$. Since we have assumed that the electrons are noninteracting and that $T \ll W$, we obtain

$$
E_{K}=2 N_{d} \int_{-W / 2}^{E_{F}} \varepsilon N(\varepsilon) d \varepsilon \equiv-2 \alpha N_{d} W N
$$

where $E_{F}$ is the Fermi energy, $N(\varepsilon)$ is the density of states per atom, orbital and spin, $N_{d}$ is the orbital degeneracy, and $\alpha$ depends on the shape of $N(\varepsilon)$ and the filling. Typically $\alpha \sim 0.1$ for a metal close to half-filling. The dependence on filling is moderate around half-filling (Calandra and Gunnarsson, 2002), but $\alpha=0$ for an empty or full band. Inserting Eq. (10) in Eq. (8) gives an approximate lower limit to the conductivity (Calandra and Gunnarsson, 2002; 2003)

$$
\sigma_{\text {sat }}(0)=\frac{\pi \alpha \gamma}{3} \frac{d^{3}}{\Omega} \frac{N_{d} e^{2}}{\hbar d}
$$

Here $\pi \alpha \gamma / 3 \sim 0.2$ depends on the details of the electronic structure, and $d^{3} / \Omega \sim 1$ depends on the lattice structure. The result is independent of $W$, since the $W$ in Eq. (8) cancels that in Eq. (10). The essential material parameters, $d$ and $N_{d}$ appear in $N_{d} e^{2} /(\hbar d)$, which has the dimension conductivity. The corresponding apparent mean free path is (Calandra and Gunnarsson, 2001)

$$
l=c N_{d}^{\frac{1}{3}} d,
$$

where $c \sim 0.6-0.8$, which is an approximate lower limit to $l$. It then follows that $l \gtrsim d$. This provides a quantummechanical derivation of the Ioffe-Regel condition, assuming that the electrons can be treated as noninteracting and that $T \ll W$. For a transition metal compound, with $N_{d}=5$ and $d \sim 3 \AA$, this leads to a resistivity of 
the order of $0.1-0.2 \mathrm{~m} \Omega \mathrm{cm}$, in agreement with the experiments.

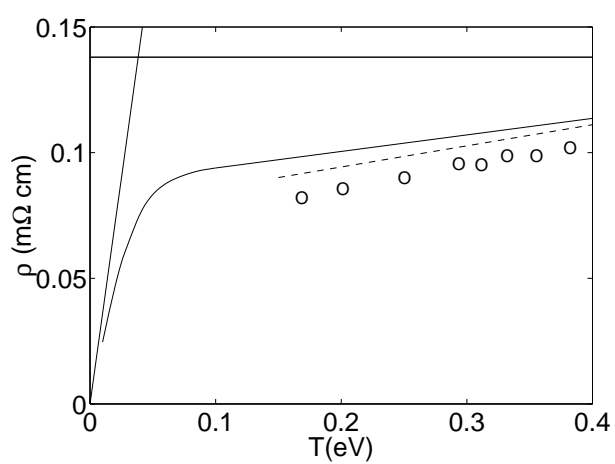

FIG. 11. Resistivity for the $\mathrm{Nb}_{3}^{*}$ model of $\mathrm{Nb}_{3} \mathrm{Sb}$, comparing the semiclassical [ broken $(N=36$ atoms $)$ and full $(N=648)$ curves] and QMC (circles, $N=36$ ) treatment of the phonons. The figure also shows the small (Eq. (13)) and large (Eq. (11)) $T$ results. It illustrates that the resistivity of the TM model saturates at large $T$ (after Calandra and Gunnarsson, 2001).

We next consider the small $T$ behavior of $\rho(T)$. In this limit the Boltzmann theory can be used, since $l \gg d$. For $T$ larger than some fraction of $\omega_{p h}$ (Grimvall, 1981)

$$
\rho(T)=8 \pi^{2} \frac{\lambda T k_{B}}{\hbar \Omega_{p l}^{2}},
$$

where $\lambda$ is the dimensionless electron-phonon coupling constant and $\Omega_{p l}$ is the plasma frequency

$$
\left(\hbar \Omega_{p l}\right)^{2}=\frac{e^{2}}{3 \pi^{2}} \sum_{\nu} \int_{B z} d^{3} k\left[\frac{\partial \varepsilon_{\nu}(\mathbf{k})}{\partial \mathbf{k}}\right]^{2} \delta\left(\varepsilon_{\nu}(\mathbf{k})-E_{F}\right) .
$$

Here $\varepsilon_{\nu}(\mathbf{k})$ is the energy of a state with the band index $\nu$ and the wave vector $\mathbf{k}$. $\Omega_{p l}$ depends on the average Fermi velocity.

The straight line corresponding to Eq. (13) is shown in Fig. 11. It shows how the conductivity is reduced and the resistivity is increased due to the reduction of the Drude peak height as $T$ is increased. The horizontal line in Fig. 11 corresponds to the large $T$ limit (Eq. (11)) of the conductivity. When this horizontal line is crossed by the steep line (Eq. (13)), the Drude peak has been so strongly reduced that it disappears under the incoherent part, and is spread out over a large energy range. As discussed above, due to the f-sum rule, the conductivity cannot normally be much further reduced at this point.

Saturation requires that the two lines (Eqs. $(11,13)$ ) cross in the available temperature range. For most metals, the line in Eq. (13) has such a small slope that the crossing would happen far above the melting point.

The model of $\mathrm{Nb}$ shows a much weaker saturation than the $\mathrm{Nb}_{3}^{*}$ model of $\mathrm{Nb}_{3} \mathrm{Sb}$ (Calandra and Gunnarsson, 2001), as is also found experimentally (see Fig. 1). This is due to a much larger $\Omega_{p l}$ for $\mathrm{Nb}(9.5 \mathrm{eV}$ in the model) than for $\mathrm{Nb}_{3} \mathrm{Sb}(3.4 \mathrm{eV})$. The slope of the line in Eq. (13) is therefore a factor of five larger for $\mathrm{Nb}_{3} \mathrm{Sb}$ than for $\mathrm{Nb}$. This leads to the much more pronounced saturation for $\mathrm{Nb}_{3} \mathrm{Sb}$. The difference in $\Omega_{p l}$ is due to the large unit cell of $\mathrm{Nb}_{3}^{*}$, which leads to many bands and many forbidden crossings. This results in quite flat bands and small electron velocities and a small $\Omega_{p l}$ (Eq. (14)). In this context it is interesting to note that $\alpha-\mathrm{Mn}$ has the most pronounced saturation among the transition metals (see Fig. 5a). The unit cell of $\alpha$-Mn has 58 atoms, suggesting a very small $\Omega_{p l}$ and a very steep initial slope of $\rho(T)$. Chakraborty and Allen (1979) and Allen and Chakraborty (1981) observed that saturation usually happens for systems with important interband transitions. Such systems typically have large unit cells and small $\Omega_{p l}$. Their observation can then be understood in terms of the arguments above.

For a one-band model with one $s$-state per unit cell, $\rho_{\text {sat }}$ is large $\left(N_{d}=1 \mathrm{in} \mathrm{Eq.} \mathrm{(11)} \mathrm{instead} \mathrm{of} N_{d}=5\right.$ for the TM model) and $\lambda / \Omega_{p l}$ is typically small. The crossing of the two lines in Eqs. $(11,13)$ then happens for such large vibration amplitudes that a calculation is not very meaningful. The main reason is that for an $s$-band model, the matrix elements do not change sign as the atoms vibrate, and very large vibration amplitudes are needed to make the scattering so strong that the Drude peak essentially disappears. It is therefore interesting to study a oneband model where the state has many strongly directed lobes. Even moderate atomic vibrations then change the signs of the matrix elements. As a model we use one of the three $t_{1 u}$ orbitals of a $\mathrm{C}_{60}$ molecule (hypothetical case of a strong crystal-field splitting). This orbital has $l=5$ and correspondingly many nodes. The resulting resistivity saturation is moderate, with the slope at large $T$ being reduced by about a factor of three to four in the semiclassical treatment of the phonons. This illustrates that interband transitions are not indispensable for resistivity saturation.

The optical conductivity in Figs. 8 and 9 can be viewed as a Drude peak riding on top of a broad structure of incoherent contributions. According to the f-sum rule and the arguments above, the height and shape of the latter part does not change much for values of $T$ discussed here. We then approximately have

$$
\sigma(\omega=0, T)=\sigma_{\text {Drude }}(\omega=0, T)+\sigma_{\text {sat }}
$$

where $\sigma_{\text {Drude }}(\omega=0, T)$ is the inverse of the resistivity in Eq. (13) and $\sigma_{\text {sat }}$ is the height of the incoherent contribution (Eq. (11)). This is the parallel resistor formula (Wiesmann et al., 1977). 


\section{STRONGLY CORRELATED SYSTEMS. HIGH- $T_{c}$ CUPRATES}

In the derivation of the Ioffe-Regel condition, we assumed i) noninteracting electrons. We now focus on the high- $T_{c}$ cuprates, where this assumption is invalid. The transport properties in the $\mathrm{CuO}_{2}$ plane are mainly determined by the antibonding band of $\mathrm{Cu} x^{2}-y^{2}$ and $\mathrm{O} 2 p$ character. It is then natural to consider one $\left(x^{2}-y^{2}\right)$ orbital per site and the orbital degeneracy $N_{d}=1$. These orbitals are put on a two-dimensional square lattice, describing the $\mathrm{CuO}_{2}$ plane. The Coulomb interaction is described by a Hubbard $U$ acting between two electrons on the same site. $U$ is assumed to be so large that states with double occupancy on a site can be projected out, leading to the $t-J$ model (Zhang and Rice, 1988).

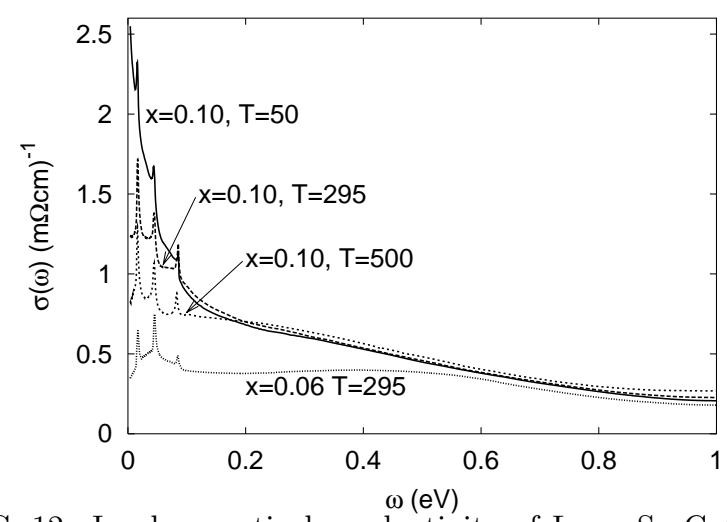

FIG. 12. In-plane optical conductivity of $\mathrm{La}_{2-x} \mathrm{Sr}_{x} \mathrm{CuO}_{4}$ as a function of $\omega$ for $x=0.06$ and $x=0.10$ (Takenaka et al., 2002). The figure illustrates how the peak at $\omega=0$ gradually goes away as $T$ is increased and that the intraband contribution to $\sigma(\omega)$ is reduced as $x$ is reduced. The sharp structures at small $\omega$ are due to phonons. Interband transitions become important for $\hbar \omega \gtrsim 1 \mathrm{eV}$.

To use the f-sum rule, we first make a simple estimate of the hopping energy. Due to the doping with $x$ holes per site, a given site is occupied by a hole or an electron with the probabilities $x$ and $(1-x)$, respectively. In the $t-J$ model, a hole can hop to a neighboring site only if the site is occupied by an electron. This suggests a hopping probability of the order of $x(1-x)$. Multiplying by the number of nearest neighbors (four) and the hopping integral $t$ gives a simple estimate of the hopping energy (Calandra and Gunnarsson, 2003)

$$
E_{K}=-4 t x(1-x) N,
$$

where $N$ is the number of sites. An exact diagonalization calculation for the $t-J$ model, gives a similar dependence on $x$ but a somewhat smaller prefactor of about 3.4 (Calandra and Gunnarsson, 2003).

Typically, $x \ll 1$. From Eq. (16) it then follows that the right hand side of the f-sum rule (Eq. (6)) should be approximately proportional to $x$, as is also found experimentally (Yamada et al., 1998). The constant of proportionality, extracted from Uchida et al. (1991), agrees with the calculation to within about 20-30\% (Calandra and Gunnarsson, 2003).

Fig. 12 shows $\sigma(\omega)$ of $\mathrm{La}_{2-x} \mathrm{Sr}_{x} \mathrm{CuO}_{4}$ for different $T$ and $x$ (Takenaka et al., 2002). The figure illustrates how the peak at $\omega=0$ gradually disappears as $T$ is increased and that for large $T$, apart from phonon structures, $\sigma(\omega)$ behave roughly as assumed in Fig. 10. A comparison of the curves for $x=0.06$ and $x=0.10$, shows that $\sigma(\omega)$ is much smaller for $x=0.06$ in the energy range $\omega \lesssim 1$ $\mathrm{eV}$, where the intraband contributions dominate. This illustrates the reduction of the intraband contribution to $\sigma(\omega)$ when $x$ is reduced, as assumed above. Similar results were found for $\mathrm{La}_{2-x}(\mathrm{Ca}, \mathrm{Sr})_{x} \mathrm{CaCuO}_{6+\delta}$ by Wang et al (2002).

We now analyze the resistivity in terms of the f-sum rule (Eqs. $(6,8))$. For conduction in the ab-plane of a cuprate, the factor $1 / 6$ in Eqs. $(6,8)$ should be replaced by a factor $1 / 4$, since the the system is quasi-twodimensional. Insertion of Eq. (16), but with the prefactor 3.4, in Eq. (8) gives

$$
\rho_{\mathrm{sat}}=\frac{0.07 c}{x(1-x)} \approx \frac{0.4}{x(1-x)} \mathrm{m} \Omega \mathrm{cm},
$$

where we have used distance $c=6.4 \AA$ between the $\mathrm{CuO}_{2}$ planes appropriate for $\mathrm{La}_{2-x} \mathrm{Sr}_{x} \mathrm{CuO}_{4}$. For small $x$, the saturation resistivity in Eq. (17) is very much larger than the Ioffe-Regel resistivity, $0.7 \mathrm{~m} \Omega \mathrm{cm}$. It is also much larger than the result in Sec. VI for weakly correlated transition metal compounds (Eq. (11))

$$
\rho_{\mathrm{sat}} \sim \frac{0.14 d}{N_{d}} \approx \frac{0.4}{N_{d}} \mathrm{~m} \Omega \mathrm{cm},
$$

where $d \sim 3$ is expressed in $\AA$. This is partly due to the degeneracy being just $N_{d}=1$ for the $t-J$ model but $N_{d}=5$ for the TM model. Furthermore, the strong correlation drastically reduces the hopping energy in the cuprates, which gives the factor $x(1-x)$ in Eq. (17). Finally, the large separation of the $\mathrm{CuO}_{2}$ planes also increases $\rho$.

Fig. 13 compares the experimental results of Takagi (1992) for $\mathrm{La}_{2-x} \mathrm{Sr}_{x} \mathrm{CuO}_{4}$ with the saturation resistivity in Eq. (17). Due to the factor $1 / x$ in Eq. (17), all resistivities are multiplied by the doping $x$. For small $x, \rho_{\text {sat }}$ is then a constant. The experimental resistivity is smaller than the predicted saturation resistivity. The same conclusion is obtained for other high- $T_{c}$ superconductors (Calandra and Gunnarsson, 2003). The experimental data do therefore not demonstrate absence of saturation. On the contrary, for $\mathrm{La}_{2-x} \mathrm{Sr}_{x} \mathrm{CuO}_{4}$ with $x=0.04$ and $x=0.07$ there are signs of saturation 
where saturation is expected to occur. It is interesting that the curves $x \rho(T)$ for $x=0.04$ and $x=0.07$ fall almost on top of each other, giving a dependence $\rho \sim 1 / x$, as suggested by the arguments above. For small dopings, saturation has also been reported (Wang et al., 1996ab) for $\mathrm{Bi}_{2} \mathrm{Sr}_{2} \mathrm{Ca}_{1-x} \mathrm{Y}_{x} \mathrm{Cu}_{2} \mathrm{O}_{8+y}$ at similar values as for $\mathrm{La}_{2-x} \mathrm{Sr}_{x} \mathrm{CuO}_{4}$ (see Fig. 2).

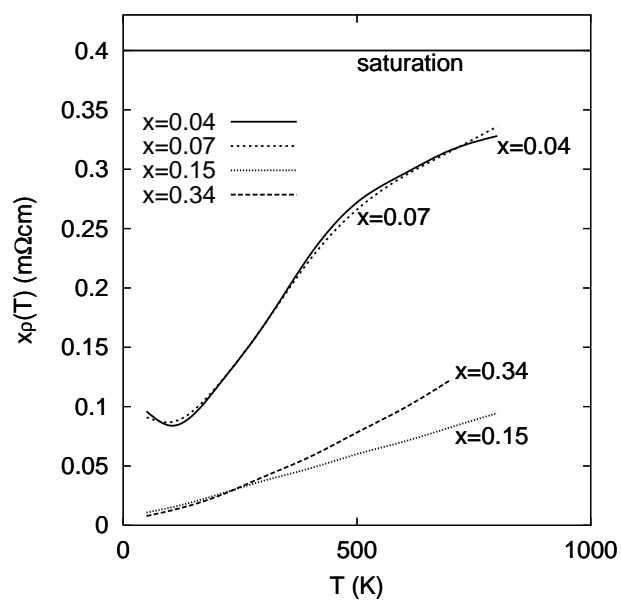

FIG. 13. Resistivity multiplied by the doping $x$ for $\mathrm{La}_{2-x} \mathrm{Sr}_{x} \mathrm{CuO}_{4}$ (Takagi et al., 1992) for $x=0.04$ (full curve), $x=0.07$ (broken curve), $x=0.15$ (dotted curve) and $x=0.34$ (chain curve). The horizontal line shows the saturation resistivity (Eq. (17)), $x \rho_{\text {sat }}$, in the limit of small $x$. The figure illustrates that the saturation resistivity is not exceeded by the experimental data and that there are signs of saturation for small $x$ roughly where saturation is expected.

Above we gave an upper limit to the resistivity without specifying a scattering mechanism. For $x=0.15$, the resistivity in the $t-J$ model is of the right order of magnitude, suggesting that electron-electron scattering can explain the resistivity, while for $x=0.07$ and $x=0.04$ the resistivity is much too small compared with experiment(Calandra and Gunnarsson, 2003). This suggests that for $x=0.04$ and $x=0.07$, there is an additional important scattering mechanism, beyond the electronelectron scattering mechanism in the $t-J$ model, or that our small cluster (16 sites) cannot describe some important scattering mechanism. This mechanism apparently increases the resistivity so rapidly for small $T$ that the upper limit (17) is approached and the resistivity shows sign of saturation. The estimate of the upper limit (17) is nevertheless correct, unless the additional scattering mechanism appreciably influences the hopping energy. The $t-J$ model alone only gives a rather weak saturation and only at larger $T$ than seen in Fig. 13.

\section{VIOLATION OF THE IOFFE-REGEL CONDITION IN OTHER COMPOUNDS}

Figs. 3 and 6 show that the Ioffe-Regel condition is violated for several other compounds. We first discuss $\mathrm{CrO}_{2}$, which is a ferromagnet below $T_{C}=390 \mathrm{~K}$. According to band structure calculations, three bands of mainly $\mathrm{Cr}_{2 g}$ spin up character are occupied by two electrons (Lewis et al., 1997; Mazin et al., 1999). We therefore consider a three-fold degenerate band with the width $W=2.3 \mathrm{eV}$ (Lewis et al., 1997) and without spin degeneracy. Fig. 9 compares the experimental $\sigma(\omega)$ at $T=300 \mathrm{~K}$ (Singley et al., 1999) with the large $T$ theoretical model in Fig. 10. The theoretical area, $\hbar^{2} \int \sigma(\omega) d \omega=2.7(\mathrm{eV})^{2}$ (for units see Appendix A) has been obtained from the fsum rule (Eq. (6)), assuming a semi-elliptical band with $W=2.3 \mathrm{eV}$. We estimate the experimental $\mathrm{t}_{2 g}$ contribution to the f-sum rule to be $2.2(\mathrm{eV})^{2}$, i.e., somewhat smaller. As a result, our estimate of the saturation resistivity $\left(\rho_{\text {sat }}=0.4 \mathrm{~m} \Omega \mathrm{cm}\right)$ is somewhat smaller than the experimental result $(\sim 0.6-0.7 \mathrm{~m} \Omega \mathrm{cm})$. The reason is probably both that the area of $\sigma(\omega)$ is somewhat overestimated and that the theoretical shape of $\sigma(\omega)$ does not take into account a strong dip in $N(\varepsilon)$ at $E_{F}$ for $\mathrm{CrO}_{2}$. These considerations suggest that theory gives a qualitatively correct description of saturation for $\mathrm{CrO}_{2}$. The strong increase in $\sigma(\omega)$ for $\omega \sim 1.5 \mathrm{eV}$ is due to interband transitions, which are neglected in the model.

This treatment assumed $\mathrm{CrO}_{2}$ to be ferromagnetic. If we had taken into account that $\mathrm{CrO}_{2}$ is paramagnetic for $T>T_{C}$, the theoretical saturation resistivity would have dropped by a factor of two, due to an increase of $\left|E_{K}\right|$. Experimentally, however, the resistivity changes little at $T=T_{C}$, suggesting that correlation effects reduce $\left|E_{K}\right|$ above $T_{C}$ in a way similar to that of magnetic effects below $T_{C}$. Actually, the Hubbard $U$ of $\mathrm{CrO}_{2}$ has been estimated to $U \approx 3 \mathrm{eV}$ (Korotin et al., 1998), comparable to the $t_{2 g}$ band width, and suggesting appreciable correlation effects. There are rather strong magnetic fluctuations in several of the systems violating the Ioffe-Regel condition. The arguments above suggest that this may reduce $\left|E_{K}\right|$ and increase the saturation resistivity.

Using similar assumptions (correlation reduces $\left|E_{K}\right|$ by a factor of two in the paramagnetic state) as for $\mathrm{CrO}_{2}$, we predict a similar saturation resistivity for $\mathrm{VO}_{2}$ and a somewhat larger value $(0.6 \mathrm{~m} \Omega \mathrm{cm})$ for $\mathrm{CaRuO}_{3}$ and $\mathrm{SrRuO}_{3}$. As for $\mathrm{CrO}_{2}$, more experimental data is needed to decide whether this agrees qualitatively with experiment. It is not clear if this framework (strong correlation effects reducing $\left|E_{K}\right|$ ) can be used to understand $\mathrm{Sr}_{2} \mathrm{RuO}_{4}$, which shows no signs of saturation up to $T=1300 \mathrm{~K}$, and $\mathrm{La}_{4} \mathrm{Ru}_{6} \mathrm{O}_{19}$, which shows signs of saturation, but at a very large resistivity. 


\section{VERY LARGE $T$ BEHAVIOR. $\mathbf{C}_{60}$ COMPOUNDS}

To derive the Ioffe-Regel condition, we assumed that ii) $T \ll W$, leading to a $T$ independent upper limit $\rho_{\text {sat }}$ to the resistivity. We now consider $T$ to be so large that the phonons substantially change $W$. Such effects are usually not very important, but the $\mathrm{C}_{60}$ model is an important exception, due to its small band width. The $T$ dependence of $\rho_{\text {sat }}$ can then be so strong that the concept of resistivity saturation becomes meaningless. In the following we neglect the electron-electron interaction, although it could play a substantial role for alkali-doped fullerides. We treat the phonons in the semiclassical approximation (Sec. IV).

For very large $T$, there is a rather trivial $T$ dependence due to the Fermi temperature, $T_{F}$, entering in the Fermi-functions of Eq. (5). Let us consider the resistivity due to static disorder. Expanding the Fermi functions in $1 / T$, we obtain $\sigma(0) \sim 1 / T$ and $\rho(T) \sim T$, although the scattering mechanism itself is $T$-independent. A similar dependence also enters for the electron-phonon scattering, which tends to mask interesting differences between couplings to the level energies (LE) and hopping integrals (HI). We therefore study the case when the Fermi functions are replaced by $\Theta$-functions in Eq. (5) $\left(T_{F}=0\right)$.

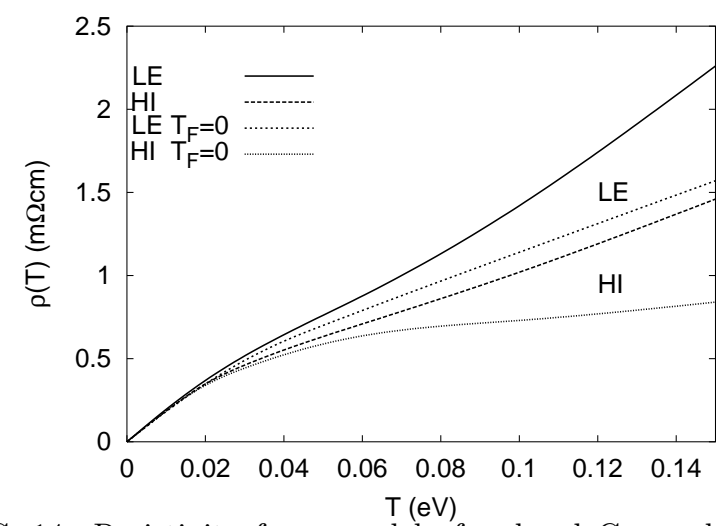

FIG. 14. Resistivity for a model of ordered $\mathrm{C}_{60}$ molecules, considering couplings to the level energies (full line, LE coupl.) and to the hopping integrals (broken line, HI coupling) in the semiclassical treatment of the phonons. Results are also shown for $T_{F}=0$, replacing the Fermi functions in Eq. (5) by $\Theta$-functions. The figure shows that there is a large difference between LE and HI coupling (after Calandra and Gunnarsson, 2002).

We consider the $\mathrm{C}_{60}$ model (see Sec. III) with either LE or HI coupling (Calandra and Gunnarsson, 2002), using the same coupling $\lambda$ in both cases (Fig. 14). While the resistivity shows no signs of saturation for the LE coupling (full curve), the model with HI coupling shows a weak saturation (broken curve), i.e., a moderate reduction of the slope for $T \gtrsim 0.03 \mathrm{eV}$. This saturation be- comes much more pronounced for $T_{F}=0$ (dotted curve). For the TM model, the slope of $\rho(T)$ is reduced at high $T$ for both HI and LE coupling, but the reduction is more pronounced for HI coupling.

To apply the analysis of Sec. V, we calculate $W(T)$ and $E_{K}(T) . W(T)$ increases with $T$ for both LE and HI coupling. In the LE case this happens because the fluctuations in the level positions increase and in the HI case because the average of the square of the hopping integrals increases. Since the average phonon amplitude squared is $\left\langle x^{2}\right\rangle \sim T$, the second moment $S_{2}(T)$ of the density of states goes as $S_{2}(T)=S_{2}(0)+a T$, where $a$ is some constant. This leads to (Calandra and Gunnarsson, 2002)

$$
W(T)=W(0) \sqrt{1+c \lambda \frac{k_{B} T}{W(T=0)}},
$$

The $T$ dependence comes in the form $\lambda T / W$, where the large prefactor $c=12$ is due to the large ratio $W^{2} / S_{2} \sim 12$. Using the considerations of Sec. VI, it follows that for the HI coupling $E_{K}$ varies in a similar way, and the $T$ dependences of $E_{K}$ and $W$ essentially cancel (Eq. (8)). This is confirmed by Fig. 14 for the $\mathrm{C}_{60}$ model (lowest HI curve), while the cancellation is less complete for the TM model due to a stronger $T$ dependence of $\alpha$ (Eq. (10)) and $\gamma$ (Eq. (8)). With LE coupling, on the other hand, $E_{K}$ is reduced as $T$ is increased. As the phonons are excited, the level energies on different sites become different and hopping becomes more difficult. For the $\mathrm{C}_{60}$ model $\left|E_{K}\right| \sim 1 / \sqrt{1+c \lambda k_{B} T / W(T=0)}$ (Calandra and Gunnarsson, 2002). For the LE coupling, the hopping energy and the band width work together, and the upper limit to the resistivity takes the form

$$
\rho_{\mathrm{sat}}(T)=\frac{0.8}{\gamma(T)}\left(1+c \lambda \frac{k_{B} T}{W(T=0)}\right) \quad \mathrm{m} \Omega \mathrm{cm}
$$

In $\mathrm{A}_{3} \mathrm{C}_{60}(\mathrm{~A}=\mathrm{K}, \mathrm{Rb})$ there is orientational disorder, i.e., the $\mathrm{C}_{60}$ molecules more or less randomly take one out of two preferred orientations (Stephens et al., 1991). As a result, the Drude peak is essentially gone already for $T=0$. The resistivity can therefore be considered as "saturated" already at $T=0$. The upper limit for the resistivity (Eq. (20)) has, however, such a strong $T$ dependence that the term "saturation" becomes meaningless in this case (see Fig. 7). The reason for this strong $T$ dependence is both the small band width and the LE coupling to the intramolecular phonons. The $T$ dependence of the TM model is weaker, due to the larger band width and to the HI coupling. Even in this case, however, $\rho(T)$ does not become a constant because of the $T$ dependence of $\alpha$ and $\gamma$. 


\section{ANDERSON METAL-INSULATOR TRANSITION AND MOTT'S MINIMUM CONDUCTIVITY.}

In the semiclassical treatment of the phonons, the phonons cause a static disorder, and the problem is therefore related to conduction in disordered system. Thus the LE and HI couplings correspond to diagonal and off-diagonal disorder, respectively. While the disordered systems are usually studied for small $T$, we are here interested in the large $T$ behavior. In the semiclassical treatment of the phonons, however, apart from causing disorder, $T$ only enters via the Fermi-functions (Eq. (5)), which is not important for the qualitative behavior.

Diagonal disorder can lead to an Anderson metalinsulator transition at $T=0$ (Lee and Ramakrishnan, 1985). For off-diagonal disorder, however, Antoniou and Economou (1977) found that there is no metal-insulator transition if the Fermi energy is located in some finite region around the middle of the band. The semiclassical calculations agree with these results, i.e., localization is found for LE but not for HI coupling as $T$ is increased.

In the QMC calculation there is no sign of localization for LE coupling, just a lack of saturation. This is natural. Localization depends sensitively on the phase factors, which are not destroyed by the elastic scattering in a disordered system. The phase information is, however, lost in the inelastic scattering by phonons at finite $T$, and localization is not expected (Lee and Ramakrishnan, 1985). These effects is properly included in the QMC but not in the semiclassical treatment of the phonons, and therfore localization shows up in the semiclassical (Calandra and Gunnarsson, 2002) but not in the QMC treatment (Gunnarsson and Han, 2000).

Mott (1974) has argued that as the disorder increases, there is a discontinuous transition from a metal to an insulator at $T=0$. He therefore introduced the concept of the minimum conductivity

$$
\sigma_{\min }=0.026 \frac{e^{2}}{\hbar d}
$$

where $d$ is the nearest neighbor atomic distance. Later work showed that the transition from a metal to an insulator actually is continuous, but that $\sigma_{\min }$ is still relevant for low but nonzero temperatures (Lee and Ramakrishnan, 1985). We therefore make a comparison of $\sigma_{\min }$ to the resistivity in the $\mathrm{TM}$ and $\mathrm{C}_{60}$ models. Converting Eq. (21) to a resistivity, we obtain

$$
\rho_{\max }=1.6 d \mathrm{~m} \Omega \mathrm{cm} \text {, }
$$

where $d$ is measured in $\AA$. Based on experiment, Mott deduced a somewhat larger minimum conductivity for systems containing transition metal atoms, resulting in the maximum resistivity

$$
\rho_{\max }=1 \mathrm{~m} \Omega \mathrm{cm} \text {. }
$$

Mott derived his result for diagonal disorder. His result can most naturally be compared with our saturation resistivity for HI coupling (off-diagonal disorder), since saturation is most pronounced in this case. The resistivity $\rho_{\max }$ is much larger than the saturation resistivity obtained above $(\mathrm{Eq} .(11,18))$ for the TM model with a five-fold degenerate orbital $\left(N_{d}=5\right)$. For a fcc lattice and a half-filled semi-elliptical band it takes the form

$$
\rho_{\mathrm{sat}}=\frac{0.14 d}{N_{d}} \mathrm{~m} \Omega \mathrm{cm},
$$

which is of the order of $0.1-0.2 \mathrm{~m} \Omega \mathrm{cm}$. The corresponding conductivity is substantially larger than Mott's minimum conductivity.

\section{CONCLUSIONS}

We have reviewed experiments showing resistivity saturation, i.e., $\rho(T)$ growing more slowly than $\rho(T) \sim T$ for large $T$. Resistivity saturation is found for several classes of metals with large resistivities, in particular for many transition metal compounds. Saturation often happens in such a way that the Ioffe-Regel condition, $l \gtrsim d$, remains fulfilled. Over the last 15 years, however, a number of metals have been found for which the Ioffe-Regel condition is violated. Some of these metals probably show resistivity saturation, but at a much larger value than the Ioffe-Regel resistivity, while in other cases little or no sign of saturation is seen.

We have reviewed early theories, presented at a time when saturation appeared to be universal. Several of the theories derived saturation. These theories emphasized different mechanisms for saturation, and no consensus was reached about which mechanism dominates.

Here we argue that it is useful to study the problem using the f-sum rule. By assuming that the (Drude) peak at $\omega=0$ is gone and that only incoherent contributions are left, we obtain an approximate upper limit, $\rho_{\text {sat }}$, to the resistivity, which usually has a weak $T$ dependence. Saturation then happens if the resistivity initially grows so rapidly that $\rho_{\text {sat }}$ is reached for small values of $T$ and if $\rho_{\text {sat }}$ has a weak $T$ dependence.

We have considered three models with qualitatively different behavior: 1) A model of weakly correlated transition metal compounds, which shows saturation in agreement with the Ioffe-Regel condition, 2) a model of strongly correlated high- $T_{c}$ cuprates, which can give saturation but at much larger values than the Ioffe-Regel resistivity, and 3) a model of alkali-doped $\mathrm{C}_{60}$ compounds, which shows no saturation.

To derive the Ioffe-Regel condition for model 1) we assumed i) noninteraction electrons and ii) $T \ll W$. Assumption i) is violated for model 2) and assumption ii) for model 3). The type of electron-phonon coupling, i.e., 
coupling to the level energies or the hopping integrals, is also important when comparing models 1) and 3).

We have focused on work where either the electronphonon or the electron-electron interaction was treated alone. In many cases this may be an oversimplification. For instance, the electron-electron interaction is believed to be important for alkali-doped fullerides (Gunnarsson, 1997), although only the electron-phonon interaction was considered here. Generally, the electron-electron interaction should reduce the magnitude of the hopping energy and tend to increase the resistivity, as was found for the high- $T_{c}$ cuprates. The interplay between the electronelectron and electron-phonon interactions may, however, be more intricate, as found for metal-insulator transitions (Han et al., 2000) and superconductivity (Han et al., 2003) in alkali-doped fullerides.

\section{APPENDIX A: MEAN-FREE PATH}

Since the mean-free path and the Ioffe-Regel resistivity play an important role in the discussion, we give details of how these quantities were obtained. The conductivity tensor can be written as (Ashcroft and Mermin, 1976)

$$
\sigma=e^{2} \sum_{\nu} \int \frac{d^{3} k}{4 \pi^{3}} \tau_{\nu}(\mathbf{k}) \mathbf{v}_{\nu}(\mathbf{k}) \mathbf{v}_{\nu}(\mathbf{k})\left[-\frac{\partial f}{\partial \varepsilon}\right]_{\varepsilon=\varepsilon_{\nu}(\mathbf{k})}
$$

where $f$ is the fermi function, $\tau_{\nu}(\mathbf{k})$ is the relaxation time and $\mathbf{v}_{\nu}(\mathbf{k})$ is the velocity for a state with band in$\operatorname{dex} \nu$ and wave vector $\mathbf{k}$. We assume a three-dimensional isotropic system and a spherical Fermi surface with one sheet. We furthermore assume that $\tau$ is independent of $\nu$ and $\mathbf{k}$. Together with $v_{F}=\hbar k_{F} / m$, where $v_{F}$ is the Fermi velocity, and $l=\tau v_{F}$, this leads to Eq. (1). Assuming that there are $M$ sheets, we find a moderate reduction of the apparent mean free path from Eq. (1) by a factor $M^{1 / 3}$. For a quasi-two-dimensional system, like the high- $T_{c}$ cuprates, we instead assume a cylindrical Fermi surface with the height $2 \pi / c$ and radius $k_{F}$, where $c$ is the average separation of the $\mathrm{CuO}_{2}$ planes. Based on photoemission results (Ino et al., 1999; Yoshida et al., 2001), we assume a "large" Fermi surface containing roughly one electron or hole (more precisely $1 \pm x$ carriers, where $x$ is the doping). This leads to $k_{F}=\sqrt{2 \pi} / a$, where $a$ is the lattice parameter of the $\mathrm{CuO}_{2}$ plane. Then

$$
\rho_{2 d}=\frac{2 \pi \hbar c}{e^{2} k_{F} l} .
$$

Assuming the Ioffe-Regel condition $l=a$, we find

$$
\rho_{2 d}^{\text {Ioffe }}=0.055\left(c / a_{0}\right) \quad \mathrm{m} \Omega \mathrm{cm}
$$

where $a_{0}=0.529 \AA$ is the Bohr radius and we have used the conversion $\hbar a_{0} / e^{2}=0.022 \mathrm{~m} \Omega \mathrm{cm}$. Assuming $c=6.4 \AA$, appropriate for $\mathrm{La}_{2-x} \mathrm{Sr}_{x} \mathrm{CuO}_{4}$, we obtain $\rho_{2 d}^{\text {Ioffe }}=0.7 \mathrm{~m} \Omega \mathrm{cm}$. If we instead had assumed a "small" Fermi surface, containing $x$ carriers, the resistivity would have been $0.7 / \sqrt{x}$. Even in this case, the experimental resistivity of $\mathrm{La}_{2-x} \mathrm{Sr}_{x} \mathrm{CuO}_{4}$ in Fig. 2 exceeds the IoffeRegel resistivity for small $x$.

For $\mathrm{A}_{3} \mathrm{C}_{60}(\mathrm{~A}=\mathrm{K}, \mathrm{Rb})$, the Ioffe-Regel resistivity was calculated by assuming that $l$ is the separation of two $\mathrm{C}_{60}$ molecules. Scattering inside the molecule is not possible at small and intermediate $T$, since this would involve scattering into states that are at least $10000 \mathrm{~K}$ higher in energy. Therefore the intermolecular separation is the appropriate length scale.

\section{REFERENCES}

Abraham, J.M., and B. Deviot, 1972, J. Less-Common Metals 29, 311 .

Allen, P.B., 1980a, in Superconductivity in $d$ - and $f$-Band Metals H. Suhl and M.B. Maple, Eds. (Academic, New York) p. 291.

Allen, P.B., 1980b, in Physics of Transition Metals, P. Rhodes, Ed. (Inst. Phys. Conf. Ser. No. 55) p. 425.

Allen, P.B., and B. Chakraborty, 1981, Phys. Rev. B 23, 4815.

Allen, P.B., R.M. Wentzcovitch, W.W. Schulz, and P.C. Canfield, 1993, Phys. Rev. B 48, 4359.

Allen, P.B., H. Berger, O. Chauvet, L. Forro, T. Jarlborg, A. Junod, B. Revaz, and G. Santi, 1996, Phys. Rev. B 53, 4393.

Antoniou, P.D., and E.N. Economou, 1977, Phys. Rev. B 16, 3768 .

Arko, A.J., F.Y. Fradin, and M.B. Brodsky, 1973, Phys. Rev. B 8, 4104.

Ashcroft, N.W., and N.D. Mermin, 1976 Solid State Physics (Holt, Rinehart and Winston, New York), p. 259.

Bass, J., 1982, in Landolt-Börnstein: Numerical data and functional relationships in science and technology, New Series III/15a, edited by K.-H. Hellwege and J.L. Olsen, (Springer, Berlin), p. 1.

Belitz, D., and W. Schirmacher, 1983, J. Phys. C: Solid State Phys. 16, 913.

Blankenbecler, R., D.J. Scalapino, and R.L. Sugar, 1981, Phys. Rev. D 24, 2278.

Calandra, M., and O. Gunnarsson, 2001, Phys. Rev. Lett. 87, 266601.

Calandra, M., and O. Gunnarsson, 2002, Phys. Rev. B 66, 205105.

Calandra, M., and O. Gunnarsson, 2003, Europhys. Lett. 61,88.

Carrington, A., D. Colson, Y. Dumont, C. Ayache, A. Bertinotti, and J.F. Marucco, 1994, Physica C 234, 1.

Chakraborty, B., and P.B. Allen, 1979, Phys. Rev. Lett. 42, 736 .

Chen, X.H., M. Yu, K.Q. Ruan, S.Y. Li, Z. Gui, G.C. Zhang, and L.Z. Cao, 1998, Phys. Rev. B 58, 14219. 
Christoph, V., and W. Schiller, 1984, J.Phys. F: Met. Khalifah, P., K.D. Nelson, R. Jin, Z.Q. Mao, Y. Liu, Q. Phys. 14, 1173.

Cote, P.J., and L.V. Meisel, 1978, Phys. Rev. Lett. 40, 1586.

Daignere, A., A. Wahl, V. Hardy, and A. Maignan, 2001, Physica C 349, 189.

Degiorgi, L., B. Briceno, M.S. Fuhrer, A. Zettl, and P. Wachter, 1994, Nature 369, 541.

Duan, H.M., R.M. Yandrofski, T.S. Kaplan, B. Dlugosch, J.H. Wang, and A.M. Hermann, 1991, Physica C 185$\mathbf{1 8 9}, 1283$.

Fisk, Z., and G.W. Webb, 1976, Phys. Rev. Lett. 36, 1084.

Fisk, Z., and A.C. Lawson, 1973, Solid State Commun. 13, 277.

Forro, L., 2002 (priv. comm.).

Girvin, S.M., and M. Jonson, 1980, Phys. Rev. B 22, 3583.

Greenwood, D.A., 1958, Proc. Phys. Soc. 71, 585.

Grimvall, G., 1981, The electron-phonon interaction in metals, North-Holland (Amsterdam) pp. 210-223.

Grimvall, G., 2001, private commun.

Grimvall, G., M. Thiessen, and A.F. Guillermet, 1987, Phys. Rev. B 36, 7816.

Guillermet, A.F. and G. Grimvall, 1991, Phys. Rev. B 44, 4332.

Gunnarsson, O., 1997, Rev. Mod. Phys. 69, 575.

Gunnarsson, O., and J.E. Han, 2000, Nature 405, 1027.

Gurvitch, M., A.K. Ghosh, B.L. Gyorffy, H. Lutz, O.F. Kammerer, J.S. Rosner, and M. Strongin, 1978, Phys. Rev. Lett. 41, 1616.

Gurvitch, M., and A.T. Fiory, 1987, Phys. Rev. Lett. 59, 1337.

Han, J.E., E. Koch, and O. Gunnarsson, 2000, Phys. Rev. Lett. 84, 1276.

Han, J.E., O. Gunnarsson, and V.H. Crespi, 2003, Phys. Rev. Lett. 90, 167006.

Hidaka, Y., and M. Suzuki, 1989, Nature 338, 635.

Hou, J.G., V.H. Crespi, X.-D. Xiang, W.A. Vareka, G. Briceno, A. Zettl, and M.L. Cohen, 1993, Solid State Commun. 86, 643.

Hou, J.G., L. Lu, V.H. Crespi, X.-D. Xiang, A. Zettl, and M.L. Cohen, 1995, Solid State Commun. 93, 973.

Imry, Y., 1980, Phys. Rev. Lett. 44, 469.

Ino, A., C. Kim, T. Mizokawa, Z.-X. Shen, A. Fujimori, M.

Takaba, K. Tamasaku, H. Eisaki, and S. Uchida, 1999, J. Phys. Soc. Jpn 68, 1496.

Ioffe, A.F., and A.R. Regel, 1960, Prog. Semicond. 4, 237. Jarrell, M., and J.E. Gubernatis, 1996, Phys. Rep. 269, 133.

Jarrell, M., and Th. Pruschke, 1994, Phys. Rev. B 49, Parcollet, O., and A. Georges, 1999, Phys. Rev. B 59, 1458.

Jonson, M., and S.M. Girvin, 1979, Phys. Rev. Lett. 43, 1447.

Hebard, A.F., T.T.M. Palstra, R.C. Haddon, and R.M. Fleming, 1993, Phys. Rev. B 48, 9945.

Nature 411, 669.

Kitazawa, K., T. Matsuura, S. Tanaka, 1981, in Ternary Superconductors, edited by G.K. Shenoy, B.D. Dunlap, and F.Y. Fradin, Elsevier, (North-Holland, New York), p. 83.

Klein, L., L. Antognazza, T.H. Geballe, M.R. Beasley, and A. Kapitulnik, 1999a, Phys. Rev. B 60, 1448.

Klein, L., L. Antognazza, T.H. Geballe, M.R. Beasley, and A. Kapitulnik, 1999b, Physica B 259-261, 431.

Kohn, W., and J.M. Luttinger, 1957, Phys. Rev. 108, 590.

Korotin, M.A., V.I. Anisimov, D.I Khomskii, and G.A. Sawatzky, 1998, Phys. Rev. Lett. 80, 4305.

Kubo, Y., Y. Shimakawa, T. Manako, T. Kondo, and H. Igarashi, 1991, Physica C 185-189, 1253.

Lange, E., and G. Kotliar, 1999, Phys. Rev. B 59, 1800.

Laughlin, R.B., 1982, Phys. Rev. B 26, 3479.

Lee, P.A., and T.V. Ramakrishnan, 1985, Rev. Mod. Phys. 57, 287.

Lewis, S.P., P.B. Allen, and T. Sasaki, 1997, Phys. Rev. B 55, 10253.

Löhle, J., J. Karpinski, A. Morawski, and P. Wachter, 1996, Physica C 266, 104.

Mahan, G.D., 1990, Many-Particle Physics, Plenum (New York), p. 651.

Mandrus, D., L. Forro, C. Kendziora, and L. Mihaly, 1992, Phys. Rev. B 45, 12640.

Marchenko, V.A., 1973, Sov. Phys.-Solid Sate 15, 1261.

Maldague, P.F., 1977, Phys. Rev. B 16, 2437.

Martin, R., K.R. Mountfield, and L. Corruccini, 1978, J. de Phys. (Paris) 39, C6-371 (1978).

Martin, S., A.T. Fiory, R.M. Fleming, L.F. Schneemeyer, and J.V. Waszczak, 1990, Phys. Rev. B 41, 846.

Mazin, I.I., D.J. Singh, and C. Ambrosch-Draxl, 1999, J. Appl. Phys. 85, 6220.

Merino, J., and R.H. McKenzie, 2000, Phys. Rev. B 61, 7996.

Millis, A.J., J. Hu, and S. Das Sarma, 1999, Phys. Rev. Lett. 82, 2354.

Mooij, J.H., 1973 phys. stat sol. (a) 17, 521.

Mott, N.F., 1974, Metal-insulator transitions, Taylor \& Francis (London).

Morton, N., B.W. James, and G.H. Wostenholm, 1978, Cryogenics 18, 131.

Orenstein, J., G.A. Thomas, A.J. Millis, S.L. Cooper, D.H. Rapkine, T. Timusk, L.F. Schneemeyer, and J.V. Waszczak, 1990, Phys. Rev. B 42, 6342. 5341.

Palstra, T.T.M., A.F. Hebard, R.C. Haddon, and P.B. Littlewood, 1994, Phys. Rev. B 50, 3462.

Pickett, W.E., K.M. Ho, and M.L. Cohen, 1979, Phys. Rev. B 19, 1734. 
Rodbell, D.S., J.M. Lommel, and R.C. DeVries, 1966, J. Phys. Soc. Japan 21, 2430.

Ron, A., B. Shapiro, and M. Weger, 1986, Phil. Mag. B 54, 553.

Ruan, K.Q., Q. Cao, S.Y. Li, G.G. Qian, C.Y. Wang, X.H. Chen, L.Z. Cao, 2001, Physica C 351, 402.

Salamon, M.B. and M. Jaime, 2001, Rev. Mod. Phys, 73, 583.

Singley, E.J., C.P. Weber, D.N. Basov, A. Barry, and J.M.D. Coey, 1999, Phys. Rev. 60, 4126.

Stephens, P.W., L. Mihaly, P.L. Lee, R.L. Whetten, S.-M Huang, R. Kaner, F. Deiderichs, and K. Holczer, 1991, Nature 351, 632.

Sunandana, C.S., 1979, J. Phys. C: Solid State Phys. 12, L165.

Sundqvist, B., and B.M. Andersson, 1990, Solid State Commun. 76, 1019

Takagi, H., B. Batlogg, H.L. Kao, J. Kwo, R.J. Cava, J.J. Krajewski, and W.F. Peck, Jr., 1992, Phys. Rev. Lett. 69, 2975.

Takenaka, K, R. Shiozaki, S. Okuyama, J. Nohara, A. Osuka, Y. Takayanagi, and S. Sugai, 2002, Phys. Rev. B 65, 092405.

Tsuei, C.C., 1986, Phys. Rev. Lett. 57, 1943.

Tyler, A.W., A.P. Mackenzie, S. NishiZaki, and Y. Maeno, 1998, Phys. Rev. B 58, R10107.

Uchida, S., T. Ido, H. Takagi, T. Arima, Y. Tokura, and S. Tajima, 1991, Phys. Rev. B 43, 7942.

Vareka, W.A., and A. Zettl, 1994, Phys. Rev. Lett. 72,
4121.

Wang, N.L., C. Geibel, and F. Steglich, 1996a, Physica C 262, 231.

Wang, N.L., B. Buschinger, C. Geibel, and F. Steglich, 1996b, Phys. Rev. B 54, 7445.

Wang, N.L., P. Zheng, T. Feng, C.D. Gu, C.C. Homes, J.M. Tranquada, B.D. Gaulin, and T. Timusk, 2002, condmat/0211224.

Weger, M., and N.F. Mott, 1985, J. Phys. C: Solid State Phys. 18, L201.

Weger, M., 1985, Phil Mag. B 52, 701.

Wiesmann, H., M. Gurvitch, H. Lutz, A. Ghosh, B. Schwartz, M. Strongin, P.B. Allen, and J.W. Halley, 1977, Phys. Rev. Lett. 38, 782.

Woodard, D.W., and G.D. Cody, 1964, Phys. Rev. 1964, A166.

Zhang, F.C., and T.M. Rice, 1988, Phys. Rev. B 37, 3759.

Yamada, K., C.H. Lee, K. Kurahashi, J. Wada, S. Wakimoto, S. Ueki, H. Kimura, Y. Endoh, S. Hosoya, G. Shirane, R.J. Birgeneau, M. Greven, M.A. Kastner, and Y.J. Kim, 1998, Phys. Rev. B 57, 6165.

Yan, S.L., Y.Y. Xie, J.Z. Wu, T. Aytug, A.A. Gapud, B.W. Kang, L. Fang, M. He, S.C. Tidrow, K.W. Kirchner, J.R. Liu, and W.K. Chu, 1998, Appl. Phys. Lett. 73, 2989.

Yoshida, T., X.J. Zhou, M. Nakamura, S.A. Kellar, P.V. Bogdanov, E.D. Lu, A. Lanzara, Z. Hussain, A. Ino, T. Mizokawa, A. Fujimori, H. Eisaki, C. Kim, Z.-X. Shen, T. Kakeshita, and S. Uchida, 2001, Phys. Rev. B 63, 220501(R). 